\title{
Nonstandard Interactions and Prospects for Studying Standard Parameter Degeneracies in DUNE and T2HKK
}

\author{
Surender Verma $(\mathbb{D}$ and Shankita Bhardwaj \\ Department of Physics and Astronomical Science, Central University of Himachal Pradesh, Dharamshala 176215, India \\ Correspondence should be addressed to Surender Verma; s_7verma@yahoo.co.in
}

Received 26 January 2019; Revised 3 April 2019; Accepted 28 April 2019; Published 13 May 2019

Academic Editor: Sally Seidel

Copyright (c) 2019 Surender Verma and Shankita Bhardwaj. This is an open access article distributed under the Creative Commons Attribution License, which permits unrestricted use, distribution, and reproduction in any medium, provided the original work is properly cited. The publication of this article was funded by SCOAP ${ }^{3}$.

\begin{abstract}
The future long baseline experiments such as DUNE and T2HKK have promising prospects to determine the neutrino mass hierarchy and measuring standard CP phase $\delta$. However, presence of possible nonstandard interactions of neutrinos with matter may intricate this picture and is the subject matter of the present work. We have studied the standard parameter degeneracies in presence of nonstandard interactions (NSI) with DUNE and T2HKK experiments. We examine the mass hierarchy degeneracy assuming (i) all NSI parameters to be nonzero and (ii) one NSI parameter $\left(\epsilon_{e \mu}\right)$ and its corresponding $C P$ phase $\left(\delta_{e \mu}\right)$ to be nonzero. We find that the latter case is more appropriate to resolve mass hierarchy degeneracy with DUNE and T2HKK experiments due to relatively small uncertainties emanating from the NSI sector. We have, also, investigated the octant degeneracy with neutrino $\left(\nu_{\mu} \longrightarrow \nu_{e}\right)$ and antineutrino $\left(\bar{v}_{\mu} \longrightarrow \bar{\nu}_{e}\right)$ mode separately. We find that to resolve this degeneracy the long baseline experiment with combination of neutrino and antineutrino mode is essential. Furthermore, we have considered DUNE in conjunction with T2HKK experiment to study $C P$ phase degeneracy due to standard $(\delta)$ and nonstandard $\left(\delta_{e \mu}\right) C P$ phases. We find that DUNE and T2HKK, in conjunction, have more sensitivity for $C P$ violation effects $(10 \sigma$ for true $\mathrm{NH}$ and $8.2 \sigma$ for true $\mathrm{IH}$ ).
\end{abstract}

\section{Introduction}

The discovery of nonzero neutrino masses and lepton flavor mixing by the reactor [1], accelerator [2], atmospheric [3], and solar [4] neutrino oscillation experiments have revealed the values of oscillation parameters such as mass squared differences $\Delta m_{21}^{2},\left|\Delta m_{31}^{2}\right|$ and mixing angles $\theta_{12}, \theta_{23}, \theta_{13}$ [5], to an unprecedented accuracy. At present, there are some unknown quantities in standard three-neutrino framework, namely, $(i)$ sign of $\Delta m_{23}^{2}$, (ii) the octant of $\theta_{23}$, and (iii) the CP-phase $\delta$, the determination of which is the prime objective of current and future neutrino oscillation experiments. The difficulty in the determination of these unknowns is the existence of degeneracies in neutrino oscillation parameters. To overcome these degeneracies, one of the methods is to combine data from different neutrino oscillation experiments. Recently, this procedure has been adopted by various studies [6-8], where the synergy between current and future experiments has been considered. In principle, future neutrino oscillation experiments have sensitivity reach to perform precision test of standard neutrino oscillation paradigm and to probe new physics beyond standard model (SM). In neutrino oscillation experiments, one model-independent way to study new physics (NP) is given by the framework of nonstandard interactions (NSI) $[9,10]$.

An alternative phenomenon to explain neutrino flavor transitions, on the basis of NSI, was first proposed by Wolfenstein [11]. Although we know that they will show their effect in neutrino oscillation experiments at subleading level, they are important, with the emergence of next generation experiments like Tokai-to-Hyper-Kamiokande (T2HK) [12], Deep Underground Neutrino Experiment (DUNE) [13], Tokai-toHyper-Kamiokande-and-Korea (T2HKK) [14], etc., where such type of interactions can be probed. In general, the NSIs may manifest itself in propagation of neutrino through matter and the processes involved in its creation and detection. The possible manifestations of NSIs have been widely studied in the literature and bounds on NSI parameters have been 
derived from various experiments $[9,10,15,16]$. Furthermore, the model-independent bounds on NSI in production and detection regions are an order of magnitude stronger than the matter NSI [15]. In this work, we focus on matter NSI which can be defined by dimension-six four-fermion operators given by $[11,17]$

$$
\mathscr{L}_{N S I}=2 \sqrt{2} G_{F} \epsilon_{\alpha \beta}^{\zeta \mathscr{K}}\left[\bar{\nu}_{\alpha} \gamma^{\rho} P_{L} \nu_{\beta}\right]\left[\bar{\zeta} \gamma_{\rho} P_{\mathscr{K}} \zeta\right]+\text { h.c. }
$$

where $\alpha, \beta=e, \mu, \tau, \mathscr{K}=L, R, \zeta=u, d, e$, and $\epsilon_{\alpha \beta}^{\zeta \mathscr{K}}$ are dimensionless parameters indicating the strength of the new interaction having units of $G_{F}$. To probe matter NSI long baseline neutrino experiments (LBNE) are ideal and the neutral current (NC) interactions which affect the neutrino propagation coherently can also be studied at far detectors. The next generation LBNE such as DUNE, T2HK, and T2HKK may reach the sensitivity to reveal NSI in neutrino sector.

In the leptonic sector, the $C P$ violation can render leptogenesis mechanism which in turn may shed light on baryogenesis $[19,20]$. It is very difficult to measure leptonic $C P$-violation in presence of NSI as it will get bewildered by the existence of possible $C P$-violation generated by NSI itself. Undoubtedly, the existence of NSI has opened an entirely new window to explore NP beyond standard model.

Previously, the authors of [6] have explored the ability to disentangle the $C P$ violating effects due to standard and nonstandard contributions under the assumption that only one NSI parameter $\epsilon_{e \mu}$ or $\epsilon_{e \tau}$ is present. In [7], the parameter degeneracies in LBNE originating from nonstandard interactions have been studied and [21] has focused on NSI at DUNE, T2HK, and T2HKK and has concluded that overall DUNE has the best sensitivity to the magnitude of the NSI parameters, while T2HKK has the best $C P$ violation sensitivity with or without NSI. Furthermore, in [22], the authors have studied the impact of nonzero NSI on the CP precision of DUNE. The authors of [23-25] have explored the effects of NSI on $C P$ violation sensitivity and hierarchy sensitivity at DUNE, respectively. In [8] the authors have studied the sensitivity to mass hierarchy, the octant of $\theta_{23}$, and $C P$ phase $\delta$ in the future long baseline experiments T2HK and DUNE assuming standard interactions (SI) only. In general, earlier studies on standard parameters degeneracies with SI or matter NSI have mostly focused on DUNE [23-26]. Motivated by the long baseline of T2HKK experiment, it is imperative to study physics potential of T2HKK and DUNE+T2HKK, in resolving standard parameter degeneracies in presence of NSI. In the present work, we have investigated prospects for lifting mass hierarchy degeneracy (sign degeneracy), $\theta_{23^{-}}$ octant degeneracy, and $C P$-phase degeneracy in DUNE, T2HKK, and DUNE+T2HKK with matter NSIs.

$\mathrm{T} 2 \mathrm{HKK}$ is a long baseline experiment proposed to enhance the hierarchy sensitivity of T2HK by setting one of the two tanks of HK detector at a site in Korea. This multidetector setup is advantageous as it gives access to a longer baseline of $1100 \mathrm{~km}$ and simultaneously boosts the data at the T2HK with baseline of $295 \mathrm{~km}$ [27]. The neutrino oscillation probabilities are strongly affected by the matter effects in long baseline experiments. These matter effects can be beneficial in lifting up the standard parameter degeneracies. Therefore, we have considered the T2HKK setup with larger baseline of $1100 \mathrm{~km}$ in the analysis. In present work, we have studied the standard parameter degeneracies, i.e., mass hierarchy degeneracy and octant degeneracy in presence of matter NSI with DUNE and T2HKK experiment. We have assumed all NSI parameters to be nonzero in one case and only one offdiagonal NSI parameter $\epsilon_{e \mu}$ to be nonzero, in another case. We find that the latter case is better at resolving standard parameter degeneracies in case of both DUNE and T2HKK experiments. Due to the larger baseline, T2HKK is found to have similar sensitivity as DUNE experiment to resolve standard parameter degeneracies including NSI. Furthermore, we have investigated the $C P$ phase degeneracy occurring due to the contribution from standard and nonstandard $C P$ phases. We observe that it is difficult to disentangle the $C P$ effects due to SI phase $\delta$ from NSI phase $\delta_{e \mu}$ at DUNE+T2HKK experiment as this conjunction is more sensitive to study $C P$ violation effects [27].

We organize the paper as follows: in Section 2, we present the formalism to write oscillation probability in presence of matter NSIs. We discuss about the long baseline experiments DUNE, T2HKK, and corresponding simulation details in Section 3. In Section 4, we discuss the prospects to resolve standard parameter degeneracies in these LBNEs. We have presented our results and, subsequent, discussion in Section 5. Finally, we conclude in Section 6.

\section{Formalism: Oscillation Probabilities}

The Hamiltonian for the neutrino propagation in presence of matter NSI can be written as

$$
H=\frac{1}{2 E}\left[U \operatorname{diag}\left(0, \Delta m_{21}^{2}, \Delta m_{31}^{2}\right) U^{\dagger}+V\right]
$$

where $U$ is the PMNS mixing matrix containing three mixing angles $\left(\theta_{i j}, i<j=1,2,3\right)$ and one $C P$ phase $\delta, \Delta m_{j i}^{2} \equiv m_{j}^{2}-$ $m_{i}^{2} . V$ is the matter potential due to interaction of neutrino with matter, viz.,

$$
\mathrm{V}=\mathscr{A}\left(\begin{array}{ccc}
1+\epsilon_{e e} & \epsilon_{e \mu} e^{i \delta_{e \mu}} & \epsilon_{e \tau} e^{i \delta_{e \tau}} \\
\epsilon_{e \mu} e^{-i \delta_{e \mu}} & \epsilon_{\mu \mu} & \epsilon_{\mu \tau} e^{i \delta_{\mu \tau}} \\
\epsilon_{e \tau} e^{-i \delta_{e \tau}} & \epsilon_{\mu \tau} e^{-i \delta_{\mu \tau}} & \epsilon_{\tau \tau}
\end{array}\right)
$$

where $\mathscr{A} \equiv 2 \sqrt{2} G_{F} N_{e}(r) E$. The unit contribution in the first element of the matrix $V$ is due to the matter term contribution from standard charged-current interactions. The diagonal element of $V$ is real, i.e., $\delta_{\alpha \beta}=0$ (where $\alpha, \beta=e, \mu, \tau$ ) for $\alpha=$ $\beta$ and $\epsilon_{\alpha \beta} \equiv \sum_{\zeta, \mathscr{K}} \epsilon_{\alpha \beta}^{\zeta \mathscr{K}}\left(N_{\zeta} / N_{e}\right)$. The oscillation probability for $\mu \longrightarrow e$ channel can be written as [7]

$$
\begin{aligned}
& P\left(v_{\mu} \longrightarrow v_{e}\right)=p^{2} f^{2}+2 p q f g \cos (\Delta+\delta)+q^{2} g^{2} \\
& \quad+4 \widehat{A} \epsilon_{e \mu}\left\{p f \left[s_{23}^{2} f \cos \left(\delta_{e \mu}+\delta\right)\right.\right.
\end{aligned}
$$




$$
\begin{aligned}
& \left.+c_{23}^{2} g \cos \left(\Delta+\delta+\delta_{e \mu}\right)\right]+q g\left[c_{23}^{2} g \cos \delta_{e \mu}\right. \\
& \left.\left.+s_{23}^{2} f \cos \left(\Delta-\delta_{e \mu}\right)\right]\right\} \\
& +4 \widehat{A} \epsilon_{e \tau} s_{23} c_{23}\left\{p f \left[f \cos \left(\delta_{e \tau}+\delta\right)\right.\right. \\
& \left.-g \cos \left(\Delta+\delta+\delta_{e \tau}\right)\right]-q g\left[g \cos \delta_{e \tau}\right. \\
& \left.\left.-f \cos \left(\Delta-\delta_{e \tau}\right)\right]\right\}+4 \widehat{A}^{2} g^{2} c_{23}^{2}\left|c_{23} \epsilon_{e \mu}-s_{23} \epsilon_{e \tau}\right|^{2} \\
& +4 \widehat{A}^{2} f^{2} s_{23}^{2}\left|s_{23} \epsilon_{e \mu}+c_{23} \epsilon_{e \tau}\right|^{2}+8 \widehat{A}^{2} f g s_{23} c_{23}\left\{c_{23}\right. \\
& \cdot \cos \Delta\left[s_{23}\left(\epsilon_{e \mu}^{2}-\epsilon_{e \tau}^{2}\right)+2 c_{23} \epsilon_{e \mu} \epsilon_{e \tau} \cos \left(\delta_{e \mu}-\delta_{e \tau}\right)\right] \\
& \left.-\epsilon_{e \mu} \epsilon_{e \tau} \cos \left(\Delta-\delta_{e \mu}+\delta_{e \tau}\right)\right\}+\mathcal{O}\left(s_{13}^{2} \epsilon, s_{13} \epsilon^{2}, \epsilon^{3}\right),
\end{aligned}
$$

$$
\begin{aligned}
& p \equiv 2 s_{13} s_{23}, \\
& q \equiv 2 r s_{12} c_{12} c_{23}, \\
& r=\left|\frac{\Delta m_{21}^{2}}{\Delta m_{31}^{2}}\right| \\
& (f, \bar{f}) \equiv \frac{\sin \left[\Delta\left(1 \mp \widehat{A}\left(1+\epsilon_{e e}\right)\right)\right]}{\left(1 \mp \widehat{A}\left(1+\epsilon_{e e}\right)\right)} \\
& g \equiv \frac{\sin \left(\widehat{A}\left(1+\epsilon_{e e}\right) \Delta\right)}{\widehat{A}\left(1+\epsilon_{e e}\right)}, \\
& \Delta \equiv\left|\frac{\Delta m_{31}^{2} L}{4 E}\right| \\
& \widehat{A} \equiv\left|\frac{A}{\Delta m_{31}^{2}}\right|
\end{aligned}
$$

where $s_{i j}=\sin \theta_{i j}, c_{i j}=\cos \theta_{i j}, i<j,(i, j)=1,2,3$. Similar expression can be obtained for inverted hierarchy (IH) by replacing $\Delta m_{31}^{2} \longrightarrow-\Delta m_{31}^{2}$ (i.e., $\Delta \longrightarrow-\Delta, \widehat{A} \longrightarrow-\widehat{A}, f \longrightarrow$ $-\bar{f}, g \longrightarrow-g$ and $q \longrightarrow-q)$. The expression for antineutrino oscillation probability can be written by replacing $\widehat{A} \longrightarrow$ $-\widehat{A}, \delta \longrightarrow-\delta$ and $\delta_{\alpha \beta}=-\delta_{\alpha \beta}$ in (4).

\section{Experimental Setups}

Considering the sensitivity reach of the present and future long baseline neutrino oscillation experiments (for example, DUNE and T2HKK), it is very important to study the individual and collective effects of NSI parameters on parameter degeneracies. We have used GLoBES package [28, 29] with best-fit values and ranges of the standard neutrino mixing parameters, as given in [30] to simulate the DUNE and T2HKK. The current bounds on NSI parameters used in present analysis are $\epsilon_{e e}<4.2,\left|\epsilon_{e \mu}\right|<0.33,\left|\epsilon_{e \tau}\right|<3.0, \epsilon_{\mu \mu}<$ $0.068,\left|\epsilon_{\mu \tau}\right|<0.04, \epsilon_{\tau \tau}<0.15[15]$. The CP phases $\delta_{\alpha \beta}$ of the off-diagonal NSI parameters are still unconstrained and can lie in the range $\delta_{\alpha \beta} \in[-\pi,+\pi]$.
The experimental configurations, energy resolutions, and systematic uncertainties considered in the present work are as follows.

3.1. DUNE. The DUNE experiment [13], situated in the USA, is a globally synchronized endeavor of neutrino physicists around the world. Out of many others, the neutrino physics goals of the experiment are to unravel the sign of neutrino mass hierarchy $\left(\Delta m_{31}^{2}\right)$ and to measure the $C P$ phase(s). The experiment is planned to direct neutrino beam from Fermilab to Homestake mine in South Dakota providing an optimum baseline of $1300 \mathrm{~km}$ for manifestation of matter effects in neutrino oscillations. Unlike Hyper-K, DUNE is an on-axis experiment. We have used DUNE CDR [13, 31] with $35 \mathrm{kt}$ LAr far detector. The optimized beam design that employs $80 \mathrm{GeV}$ beam of protons having 1.0 MW power has been used to simulate the experiment. We have considered $5(+5)$ years of run in neutrino (antineutrino) mode resulting in an exposure of 350 kt.MW.years. The appearance efficiency $\left(\epsilon_{a p p}\right)$ and energy resolutions $\left(E_{R_{e}}, E_{R_{\mu}}\right)$ taken in the present analysis are $80 \%(0.15 / \sqrt{E}, 0.2 / \sqrt{E})$, respectively. The normalization and energy calibration uncertainty for $\nu_{e}$ signal $\left(N_{S}, E_{S}\right)$ /background $\left(N_{B}, E_{B}\right)$ is taken to be $N_{S}=5 \%$, $E_{S}=2 \%, N_{B}=10 \%$, and $E_{B}=10 \%$. For $\nu_{\mu}$ signal $\left(N_{S}, E_{S}\right)$ /background $\left(N_{B}, E_{B}\right)$ the values are $N_{S}=5 \%, E_{S}=$ $5 \%, N_{B}=10 \%$, and $E_{B}=10 \%$.

3.2. T2HKK. The T2HKK experiment [14], an extension T2HK [12], is proposed to be stretched over Japan and Korea. The neutrino beam will be directed from J-PARC facility in Japan to two water-Cherenkov detectors: (i) first detector at Kamioka mine in Japan with a baseline of $295 \mathrm{~km}$; (ii) second detector to be built in Korea providing a baseline of $1100 \mathrm{~km}$. In the present work, we have considered 1100 $\mathrm{km}$ baseline (also, referred to as T2HKK), with detector at $1.5^{\circ}$ off-axis with respect to the neutrino beam, where matter effects will be large. We choose 13 MW.years beam power which is similar to that of $\mathrm{T} 2 \mathrm{HK}$. The running time, in ratio 1:3 for neutrino and antineutrino mode, is 10 years amounting to total exposure of $2.7 \times 10^{22}$ protons on target (POT). The appearance efficiency $\left(\epsilon_{a p p}\right)$ and energy resolutions $\left(E_{R_{e}}, E_{R_{\mu}}\right)$ taken in the present analysis are $50 \%(0.085 / \sqrt{E}, 0.085 / \sqrt{E})$, respectively. The normalization and energy calibration uncertainty for $\nu_{e} \operatorname{signal}\left(N_{S}, E_{S}\right) /$ background $\left(N_{B}, E_{B}\right)$ are taken to be $N_{S}=5 \%, E_{S}=0.01 \%, N_{B}=5 \%$, and $E_{B}=0.01 \%$. For $\nu_{\mu} \operatorname{signal}\left(N_{S}, E_{S}\right) /$ background $\left(N_{B}, E_{B}\right)$ the values are $N_{S}=$ $2.5 \%, E_{S}=0.01 \%, N_{B}=20 \%$, and $E_{B}=0.01 \%$.

\section{Parameter Degeneracies}

In general, different set of oscillation parameters may give identical predictions for oscillation probability resulting in parameter degeneracies and making it difficult to uniquely determine these parameters. The biprobability plots are well known constructions to study parameter degeneracies in presence of NSI parameters and CP phases. In presence of off-diagonal NSI the neutrino/antineutrino oscillation probability exhibits degeneracy between SI and NSI phase when 


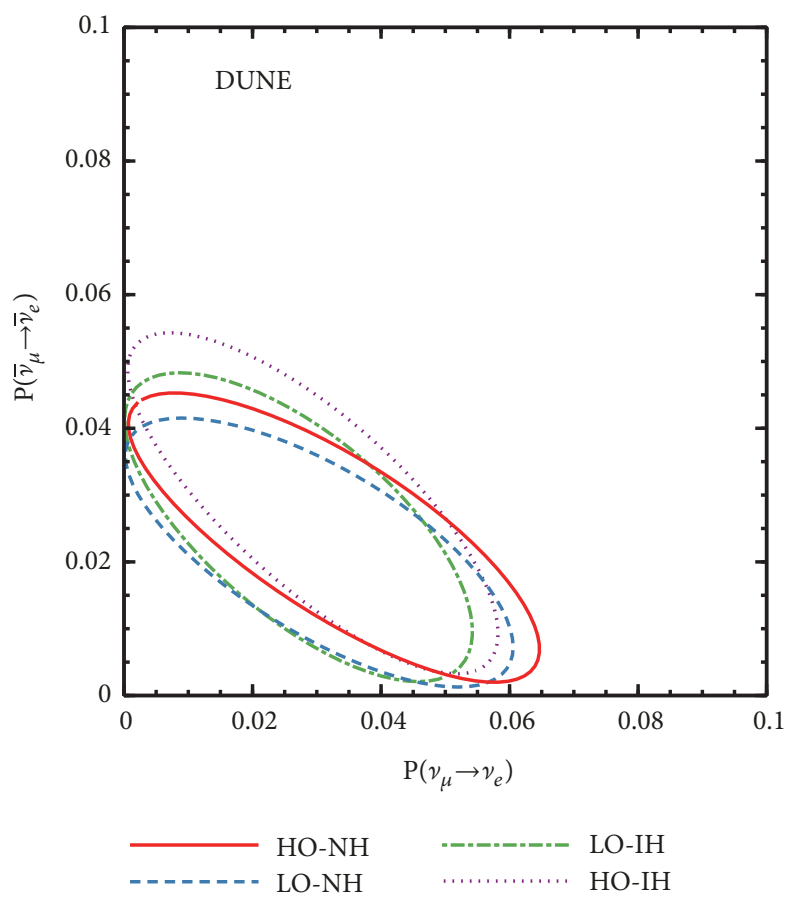

Figure 1: The biprobability plots with $\epsilon_{e \mu}=0.04$ and $C P$-phase $\delta_{e \mu}=\pi / 2$ for DUNE with baseline $L=1300 \mathrm{~km}$ and $E=3 \mathrm{GeV}$. The standard $C P$-phase $\delta$ is varied from 0 to $2 \pi$.

$P^{S I}(\delta)=P^{N S I}\left(\phi, \epsilon_{e \mu}, \delta_{e \mu}\right)$ and $\bar{P}^{S I}(\delta)=\bar{P}^{N S I}\left(\phi, \epsilon_{e \mu}, \delta_{e \mu}\right)$, where $\phi$ is Dirac $C P$ phase in a model with NSI. If we presume that mixing angles and mass squared differences are known from some other experiment, then for every value of SI phase $\delta$ there will be three unknowns $\left(\phi, \epsilon_{e \mu}\right.$, and the phase $\delta_{e \mu}$ ) which generate an off-diagonal NSI degeneracy. Accordingly, a measurement of $P$ and $\bar{P}$ in an experiment provides two constraints; for each value of $\delta$, a solution for $\epsilon_{e \mu}$ and $\delta_{e \mu}$ will exist for any value of $\phi$ resulting in parameter degeneracy. As a representative plot to depict standard parameter degeneracies we have shown, in Figure 1, mass hierarchy and octant degeneracy assuming NSI parameters $\epsilon_{e \mu}=0.04, \delta_{e \mu}=\pi / 2$ and varying $\delta$ from 0 to $2 \pi$ for DUNE experiment. All other NSI parameters are assumed to be zero. The solid and dashed ellipses correspond to higher octant (HO) and lower octant (LO) of $\theta_{23}$, respectively, for normal hierarchy $(\mathrm{NH})$. Similarly, the dotted and dash-dotted ellipses correspond to higher octant (HO) and lower octant (LO) of $\theta_{23}$, respectively, for inverted hierarchy $(\mathrm{IH})$. The significant overlapping of the ellipses shows fourfold $\theta_{23}$ octant and mass hierarchy degeneracy. For example, points of intersection of solid (dotted) and dashed (dash-dotted) ellipses exhibit $\theta_{23}$ octant degeneracy as neutrino and antineutrino oscillation probabilities are the same in both cases for normal (inverted) hierarchy.

4.1. Mass Hierarchy Degeneracy. In the determination of unknown neutrino mixing parameters an ambiguity exists in correlated way between $\delta$ and sign of $\Delta m_{31}^{2}$. The sign of $\Delta m_{31}^{2}$ can be determined by measuring interference between the vacuum and the matter effects. The simultaneous determination of $\delta$ and sign of $\Delta m_{31}^{2}$ can be done in long baseline experiments in both standard [8] and nonstandard cases [6]. Thus, we study the prospects to resolve this degeneracy with two future long baseline experiments DUNE and T2HKK involving NSIs. Throughout this work we have considered the $v_{\mu} \longrightarrow v_{e}$ channel to study various parameter degeneracies. We have obtained plots for DUNE and T2HKK for two cases assuming (i) all NSI parameters to be nonzero, i.e., $\epsilon_{e e}=$ $0.4, \epsilon_{e \mu}=0.04, \epsilon_{e \tau}=0.04, \epsilon_{\mu \mu}=0.03, \epsilon_{\mu \tau}=0.04, \epsilon_{\tau \tau}=0.1$ and NSI CP phases $\delta_{\alpha \beta}=[-\pi, \pi]$; (ii) only $\epsilon_{e \mu}, \delta_{e \mu}$ to be nonzero $\left(\epsilon_{e \mu}=0.04, \delta_{e \mu}=[-\pi, \pi]\right)$ (Figure 2).

We have, also, shown sensitivity plots for mass hierarchy for cases (i) and (ii) in Figures 3(a) and 3(b) and Figures $3(\mathrm{c})$ and $3(\mathrm{~d})$, respectively. We plot significance $\left(\sigma=\sqrt{\chi^{2}}\right)$ as a function of $\delta$ (true) to study hierarchy sensitivity of DUNE and T2HKK experiments for true $\mathrm{NH}$ (true $\mathrm{IH}$ ) in left panel(right panel) of Figure 3 . The true $\mathrm{NH}$ (true $\mathrm{IH}$ ) sensitivity plot is obtained by considering $\mathrm{NH}(\mathrm{IH})$ in the true spectrum and $\mathrm{IH}(\mathrm{NH})$ in test spectrum. We have marginalized over $\delta, \theta_{23}$ and $\epsilon$ by considering them in test spectrum. The statistical definition of $\chi^{2}$ for understanding the aspects of the mass hierarchy sensitivity plot in case of true $\mathrm{NH}$ is as follows:

$$
\begin{aligned}
\chi_{N H}^{2} & \equiv \min _{\left(\delta, \theta_{23}, \epsilon\right)_{\text {test }}} \\
& \sum_{i=1}^{x} \sum_{j, k=1}^{2} \frac{\left[N_{N H}^{i, j, k}\left(\delta, \theta_{23}, \epsilon\right)_{\text {true }}-N_{I H}^{i, j, k}\left(\delta, \theta_{23}, \epsilon\right)_{\text {test }}\right]^{2}}{N_{N H}^{i, j, k}\left(\delta, \theta_{23}, \epsilon\right)_{\text {true }}}
\end{aligned}
$$



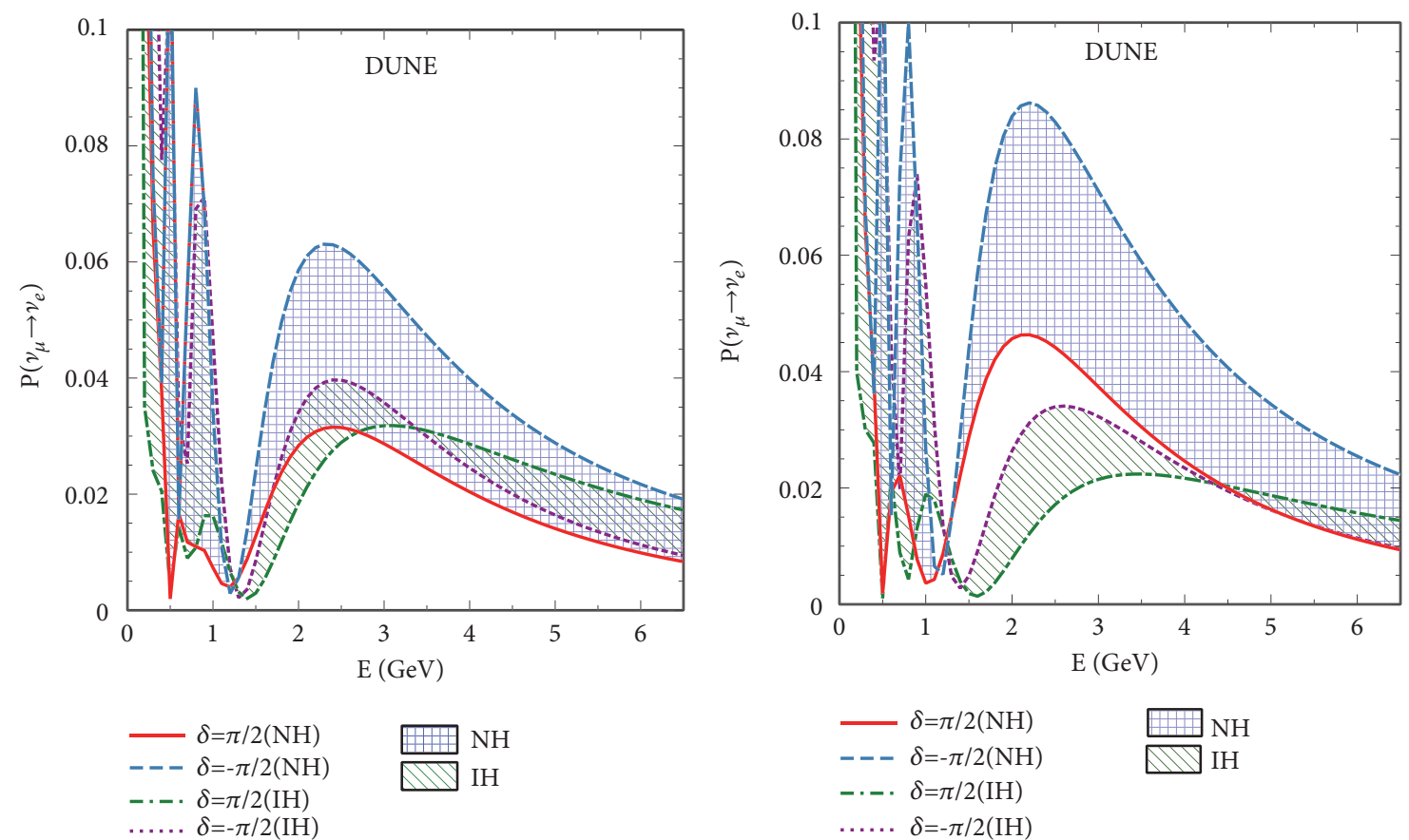

(a)

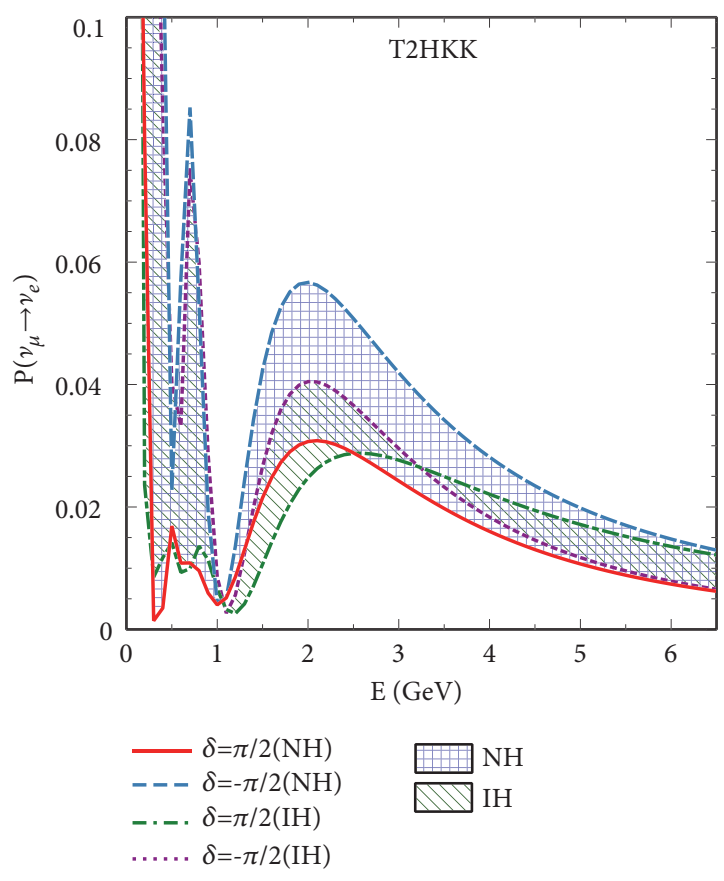

(c) (b)

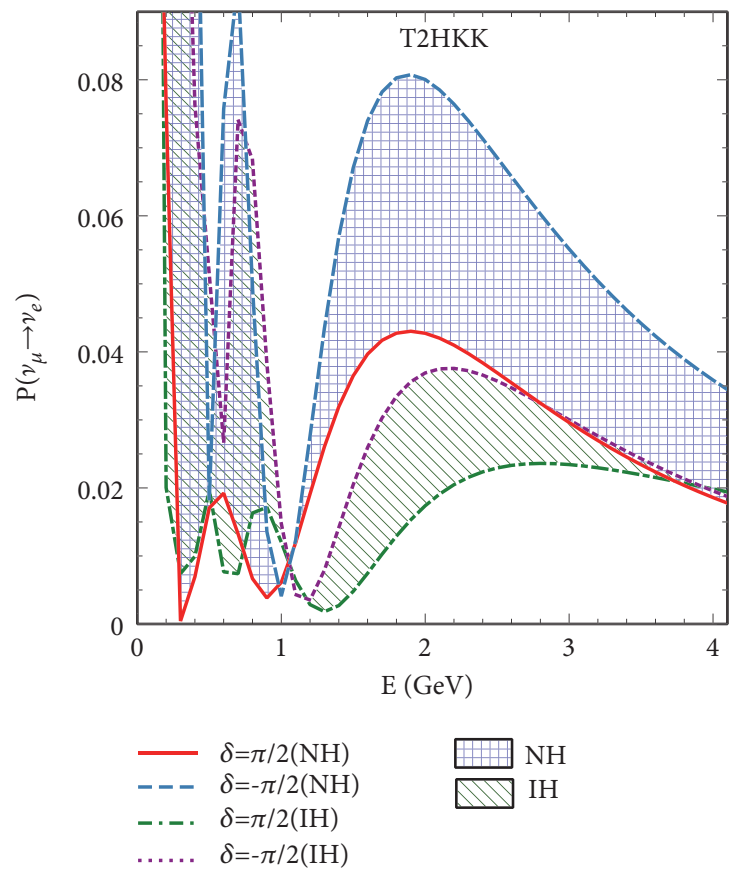

(d)

Figure 2: The appearance probability $P\left(\nu_{\mu} \longrightarrow \nu_{e}\right)$ as function of neutrino beam energy $E$ for DUNE (first row) and T2HKK (second row). The left (right) panel describes mass hierarchy assuming all $\left(\epsilon_{e \mu}, \delta_{e \mu}\right)$ NSI parameters nonzero. $\theta_{23}=42^{\circ}\left(\theta_{23}=48^{\circ}\right)$ for left panel (right panel). The band comes due to variation of $\delta, \delta_{e \mu} \in[-\pi, \pi]$ and boundaries correspond to $\delta= \pm \pi / 2$ for $\mathrm{NH}$ as well as IH.

where $N_{N H}^{i, j, k}$ and $N_{I H}^{i, j, k}$ denote the number of true events and test events for $\mathrm{NH}$ and $\mathrm{IH}$ in the $(i, j, k)^{\text {th }}$ bin, respectively. The index $i$ runs over 1 to $x ; x$ is the number of bins for particular experiment. For DUNE, $x=39$, of $250 \mathrm{MeV}$ width in $0.5-10 \mathrm{GeV}$ range and for T2HKK $x=20$, of $40 \mathrm{MeV}$ width in $0.4-1.2 \mathrm{GeV}$ range. The index $j$ describes type of mode, i.e., neutrino or antineutrino. $j=1(j=2)$ for neutrino (antineutrino) mode. The index $k$ describes type of channel considered, i.e., appearance or disappearance. $k=1(k=2)$ for appearance (disappearance) channel. The NSI parameter 


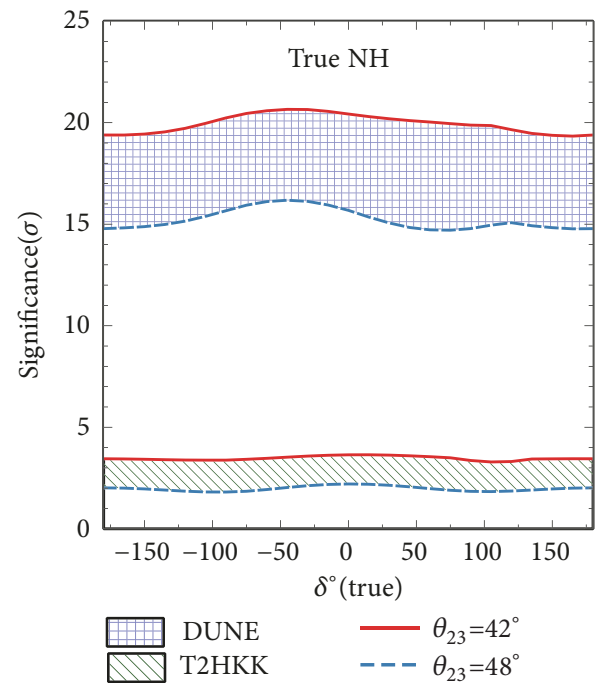

(a)

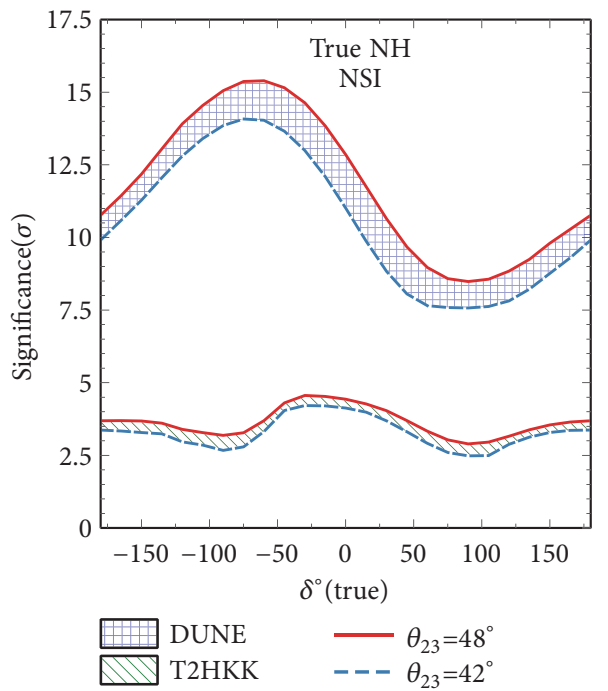

(c)

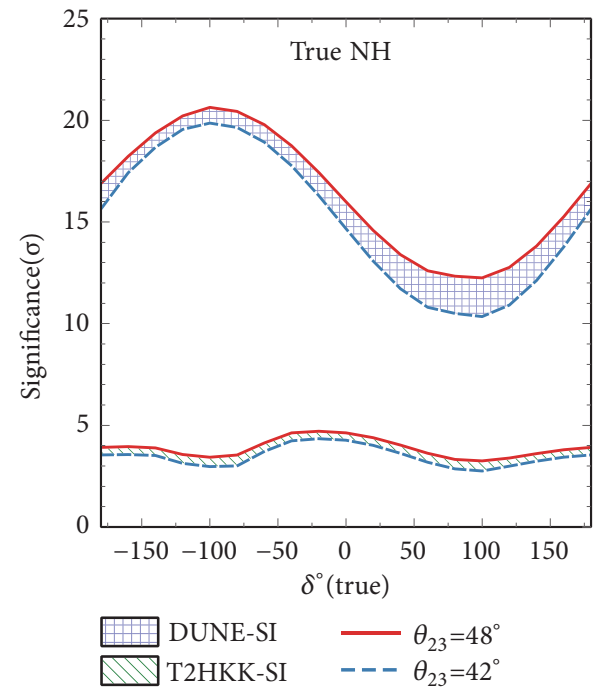

(e)

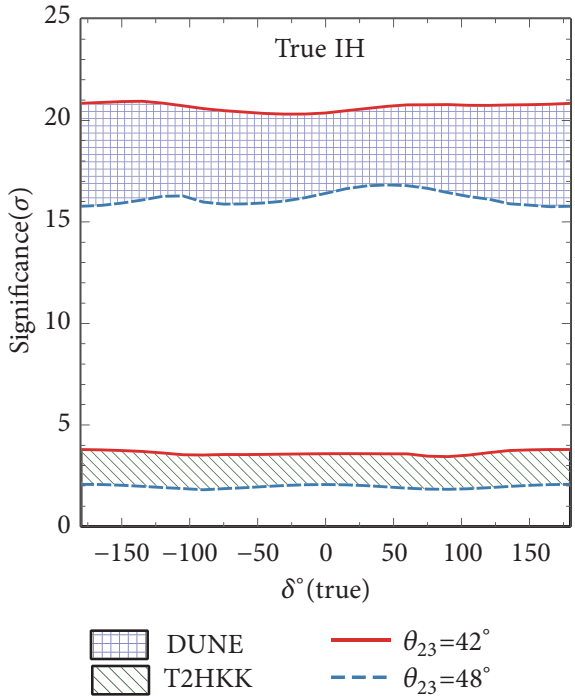

(b)

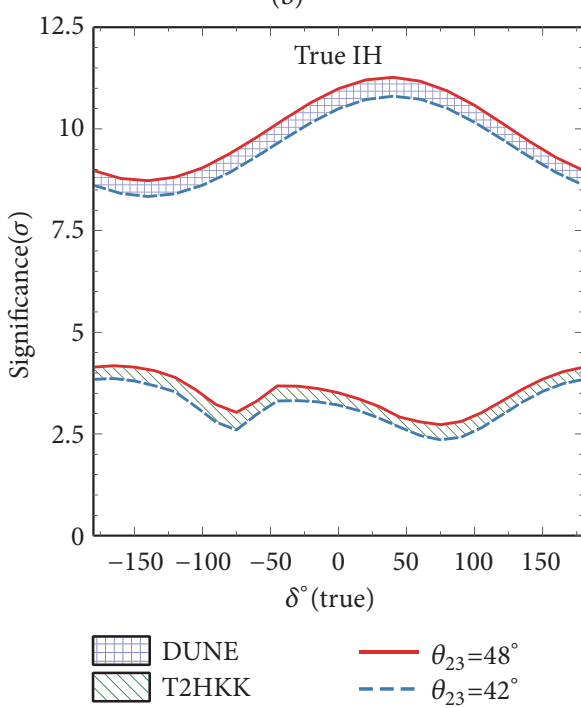

(d)

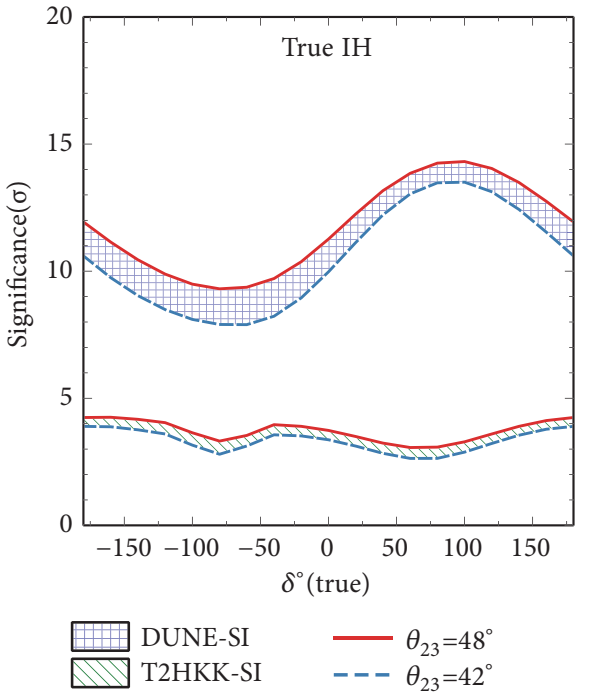

(f)

FIGURE 3: Sensitivity plots for mass hierarchy. The plots for significance $\left(\sqrt{\chi^{2}}\right)$ as a function of $\delta($ true $)$ with true NH (left panel) and true IH (right panel). The first (second) row describes mass hierarchy sensitivity assuming all $\left(\epsilon_{e \mu}, \delta_{e \mu}\right)$ NSI parameters nonzero. Figures 3(e) and 3(f) show the sensitivity plots for SI case. The band comes due to variation of $\theta_{23}$ and boundaries correspond to $\theta_{23}=42^{\circ}$ and $48^{\circ}$. 
TABLE 1: The true values and marginalization ranges for all NSI parameters used in the analysis $[15,18]$.

\begin{tabular}{|c|c|c|}
\hline NSI Parameter & True value & Marginalization range \\
\hline$\epsilon_{e e}$ & 0.4 & {$[-0.4,0.4]$} \\
\hline$\epsilon_{\mu \mu}$ & 0.03 & {$[-0.05,0.05]$} \\
\hline$\epsilon_{\tau \tau}$ & 0.1 & {$[-0.15,0.15]$} \\
\hline$\epsilon_{e \mu}$ & 0.04 & {$[0,0.10]$} \\
\hline$\epsilon_{e \tau}$ & 0.04 & {$[0,0.10]$} \\
\hline$\epsilon_{\mu \tau}$ & 0.04 & {$[0,0.04]$} \\
\hline$\delta_{e \mu}$ & {$[-\pi, \pi]$} & {$[-\pi, \pi]$} \\
\hline$\delta_{e \tau}$ & {$[-\pi, \pi]$} & {$[-\pi, \pi]$} \\
\hline$\delta_{\mu \tau}$ & {$[-\pi, \pi]$} & {$[-\pi, \pi]$} \\
\hline
\end{tabular}

$\epsilon=\epsilon_{\alpha \beta} e^{i \delta_{\alpha \beta}}$. We have minimized over the marginalization ranges of NSI parameters as given in Table 1.

4.2. Octant Degeneracy. The oscillation probabilities for disappearance channels $1-P\left(\nu_{\mu} \longrightarrow \nu_{e}\right)$ (neutrino) and $1-$ $P\left(\overline{\nu_{\mu}} \longrightarrow \overline{\nu_{e}}\right)$ (antineutrino) show main contribution from $\sin ^{2} 2 \theta_{23}$. In case, $\theta_{23}$ is not maximal, we have two possibilities: either $\cos 2 \theta_{23}>0$ or $\cos 2 \theta_{23}<0$. This ambiguity creates two solutions $\left(\theta_{23}, \delta\right)$ and $\left(90^{\circ}-\theta_{23}, \delta^{\prime}\right)$. The resolution of uncertainty in $\theta_{23}$ due to octant degeneracy is important for precise measurement of $\theta_{23}$. We study this degeneracy in both neutrino and antineutrino mode with DUNE and T2HKK assuming NSI parameters $\epsilon_{e \mu}=0.04$ and $\delta_{e \mu}=[-\pi, \pi]$ (Figure 4).

To study the $\theta_{23}$ octant degeneracy, we have obtained octant sensitivity plot for true $\mathrm{NH}$ (true $\mathrm{IH}$ ) in Figure 5. We plot significance $(\sigma)$ as a function of $\theta_{23}($ true $)$. One can define octant sensitivity by considering LO in true spectrum ( $\mathrm{HO}$ in true spectrum) and $\mathrm{HO}$ in test spectrum(LO in test spectrum). The octant sensitivity tells us about the ability of an experiment to distinguish the $\theta_{23}$ lower octant from its higher octant. In order to obtain sensitivity plots for octant degeneracy, we have marginalized over sign $\left(\Delta m_{31}^{2}\right), \delta$, and NSI parameter $\epsilon$ and the $\chi^{2}$ in true NH case is

$$
\chi_{N H}^{2} \equiv \min _{\left(\delta, \theta_{23}, \Delta m_{31}^{2}, \epsilon\right)_{t e s t}} \sum_{i=1}^{x} \sum_{j, k=1}^{2} \frac{\left[N_{N H}^{i, j, k}\left(\delta, \theta_{23}, \Delta m_{31}^{2}, \epsilon\right)_{\text {true }}-N_{N H}^{i, j, k}\left(\delta, \theta_{23}, \Delta m_{31}^{2}, \epsilon\right)_{\text {test }}\right]^{2}}{N_{N H}^{i, j, k}\left(\delta, \theta_{23}, \Delta m_{31}^{2}, \epsilon\right)_{\text {true }}},
$$

4.3. CP Phase Degeneracy. In presence of new physics, there may appear additional sources of $C P$ violation other than due to Dirac-type $C P$ phase $\delta$. The $C P$ effects will, in general, include contributions from both standard and nonstandard $C P$ phases. As the value of $C P$ phases $\delta$ and $\delta_{e \mu}$ is not known and possible existence of $C P$ violation in nature, we look for all possible values of $\delta$ (true), $\delta_{e \mu}$ (true) which are distinct from the $C P$ conserving values of $\delta$ and $\delta_{e \mu}$. For this purpose, we define $C P$ fraction [32] $F(\delta)\left(F\left(\delta_{e \mu}\right)\right)$ as the fraction of total permitted range of $\delta($ true $)\left(\delta_{e \mu}\right.$ (true $\left.)\right)$, i.e., $[-\pi, \pi]$ where $C P$ violation effects corresponding to standard(nonstandard) $C P$ phases can be explored. Also, we have excluded the $C P$ conserving values by marginalizing over $\delta($ test $)\left(\delta_{e \mu}(\right.$ test $\left.)\right)$ for $\{0, \pi\}$ which implies that the value of $\delta($ test $)\left(\delta_{e \mu}(\right.$ test $\left.)\right)$ get fixed to its $C P$ conserving values 0 or $\pi$ (Figure 6(b)). So, we can write $C P$ fraction $F(\delta)\left(F\left(\delta_{e \mu}\right)\right)$ at $3 \sigma$ C.L. = Total range of $\delta$ (true $)\left(\delta_{e \mu}\right.$ (true) $)$ values above $3 \sigma$ C.L./ Total permitted range of $\delta($ true $)\left(\delta_{e \mu}(\right.$ true $\left.)\right)([-\pi, \pi])$ which will be discussed in detail, in Section 5.

To resolve $C P$ phase degeneracy, we need to find out the $C P$ sensitivity with which an experiment can distinguish between $C P$ conserving cases and $C P$ violating cases. In standard oscillations there is only one degree of freedom in $\chi^{2}$-function corresponding to standard CP phase $\delta$. However, in case of neutrino oscillations with NSI there are two degrees of freedom due to standard and nonstandard $C P$ phases $\left(\delta, \delta_{e \mu}\right)$. We define the $\chi^{2}$-function as

$$
\equiv \min _{\delta, \epsilon_{e \mu}, \delta_{e \mu}} \sum_{i=1}^{x} \sum_{j}^{2} \frac{\left[N_{t r u e}^{i, j}\left(\delta, \epsilon_{e \mu}, \delta_{e \mu}\right)-N_{t e s t}^{i, j}\left(\delta, \epsilon_{e \mu}, \delta_{e \mu}\right)\right]^{2}}{N_{\text {true }}^{i, j}\left(\delta, \epsilon_{e \mu}, \delta_{e \mu}\right)},
$$

where $N_{\text {true }}^{i, j}$ are the number of true events for $\left(\delta, \delta_{e \mu}\right)$ in the range $[-\pi, \pi]$ and $N_{\text {test }}^{i, j}$ are the number of test events in $(i, j)^{\text {th }}$ bin for $\left(\delta, \delta_{e \mu}\right)$ with $\{0, \pi\}$.

To study $C P$ violation discovery, we obtain plot for $\chi^{2}$ as a function of $\delta$ (true) for true $\mathrm{NH}$ and true IH for DUNE, T2HKK, and DUNE+T2HKK experiments (Figure 7).

\section{Results and Discussion}

The degeneracy in sign of $\Delta m_{31}^{2}$ and $\delta$ can be resolved with the experiments which involve matter effects such as DUNE and T2HKK. In left (right) panel of Figure 2, we have shown the mass hierarchy degeneracy assuming all $\left(\epsilon_{e \mu}, \delta_{e \mu}\right)$ NSI parameters, along with corresponding $C P$ phases, to be nonzero for DUNE and T2HKK experiments. The meshed 


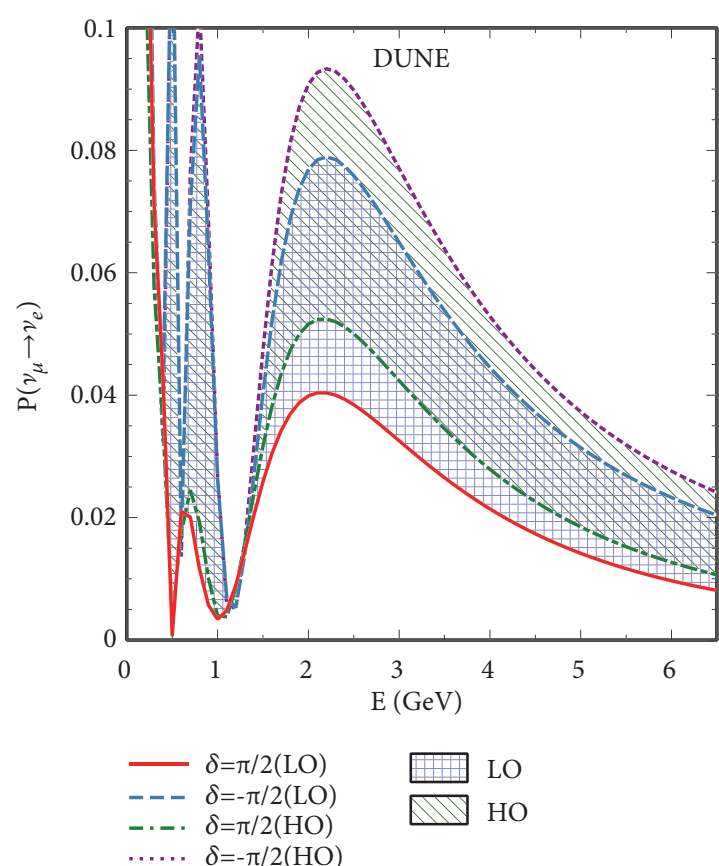

(a)

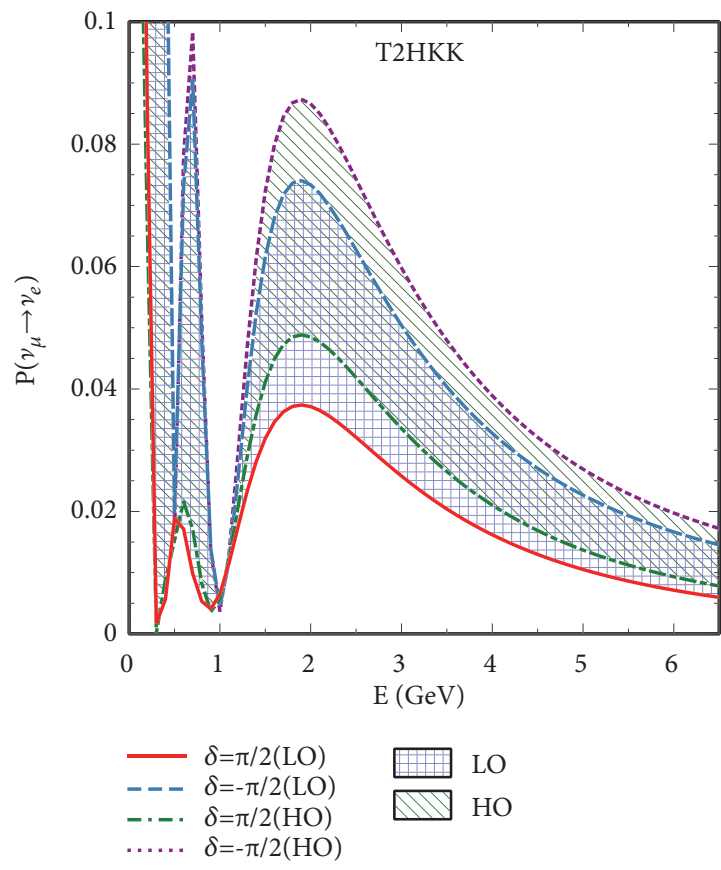

(c)

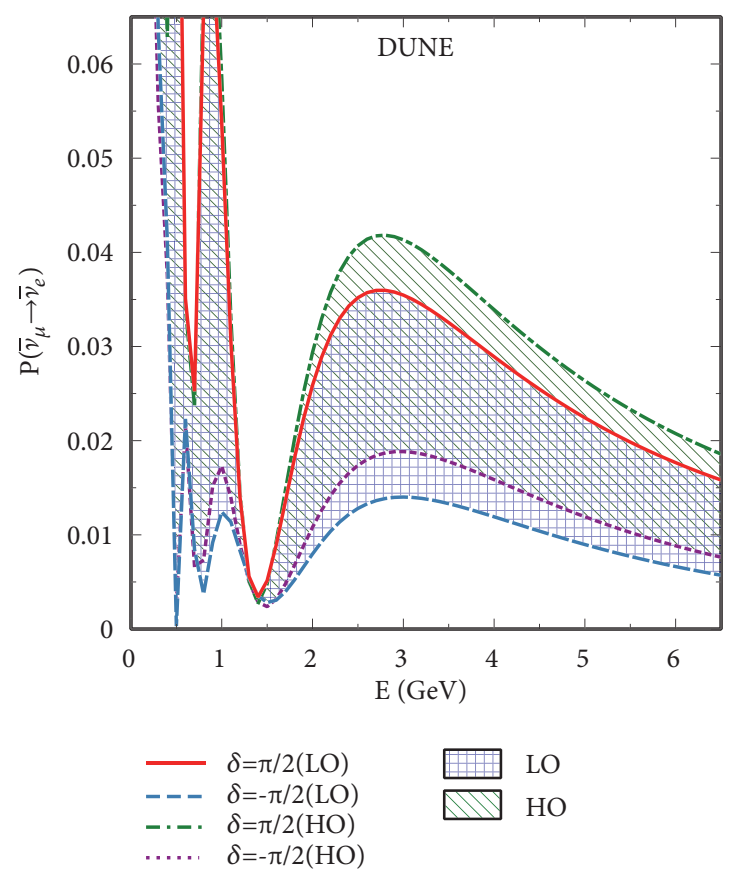

(b)

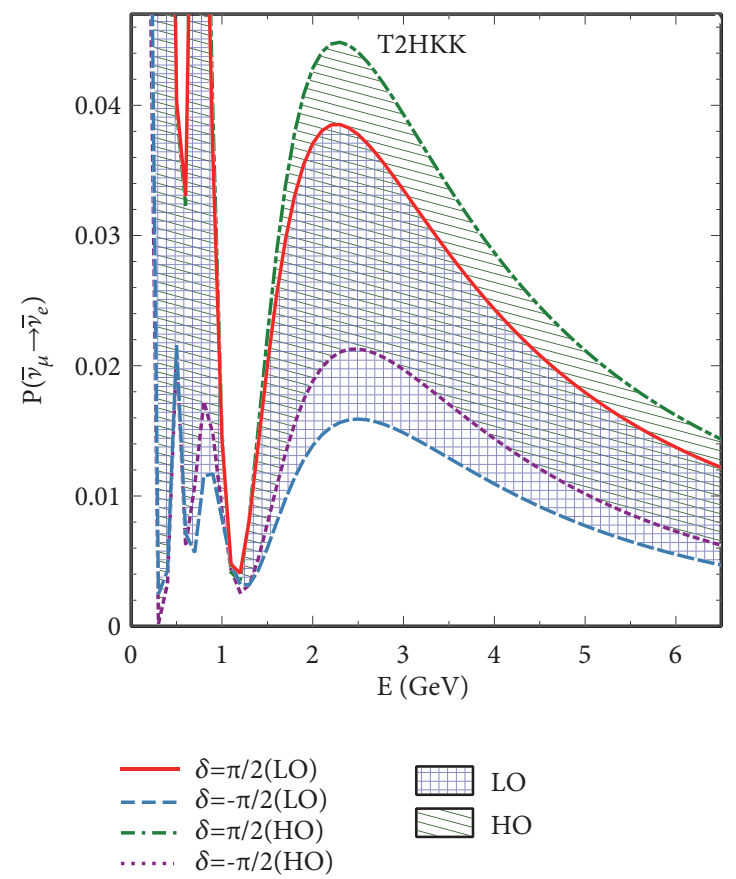

(d)

FIGURE 4: The appearance probability for neutrino (antineutrino) mode as function of neutrino beam energy $E$ for DUNE (first row) and T2HKK (second row) assuming NSI parameters $\epsilon_{e \mu}=0.04$ and $\delta_{e \mu}=[-\pi, \pi]$. The octant degeneracy is represented for neutrino mode (left panel) and for antineutrino mode (right panel). The band comes due to variation of $\delta, \delta_{e \mu} \in[-\pi, \pi]$ and the boundaries of the bands correspond to $\delta= \pm \pi / 2$ for LO and HO. The value of $\theta_{23}=42^{\circ}$ for LO and $\theta_{23}=48^{\circ}$ for $\mathrm{HO}$.

region between solid and dashed lines corresponds to the normal hierarchy $(\mathrm{NH})$ whereas the forward-diagonal region between dash-dotted and dotted lines corresponds to the inverted hierarchy (IH) of neutrino masses. The normal and inverted hierarchy regions overlap for DUNE and T2HKK experiments due to contributions from all the nonzero NSI parameters (left panel) making it difficult to resolve the mass hierarchy degeneracy in these experiments. However, there is no overlap in the $\mathrm{NH}$ and $\mathrm{IH}$ regions for DUNE and $\mathrm{T} 2 \mathrm{HKK}$ for energy range 1 to $4 \mathrm{GeV}$ assuming only 


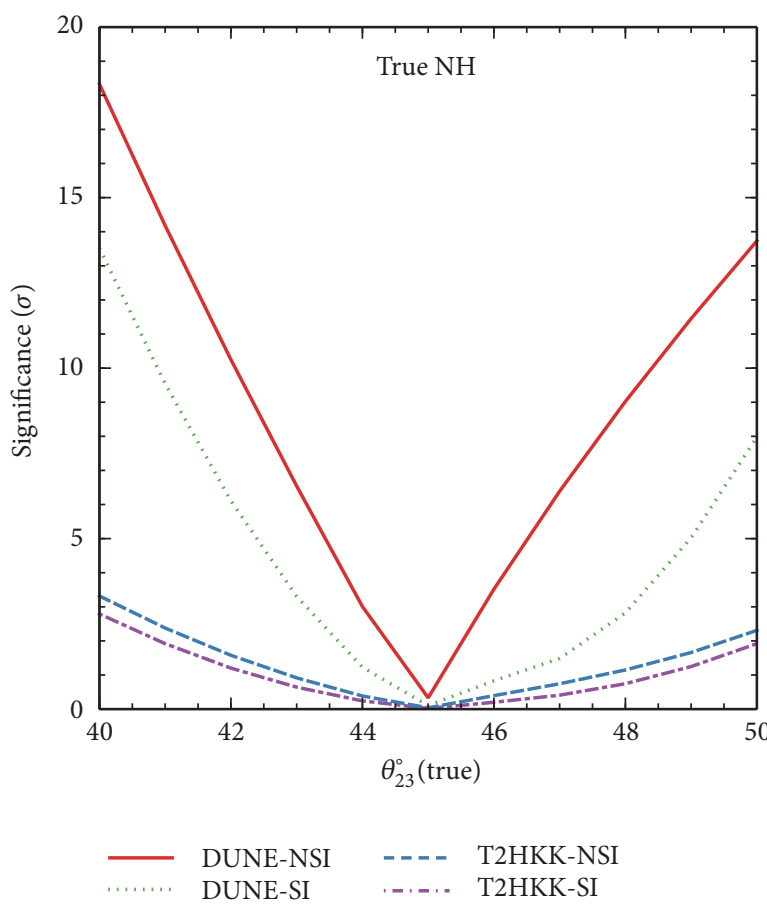

(a)

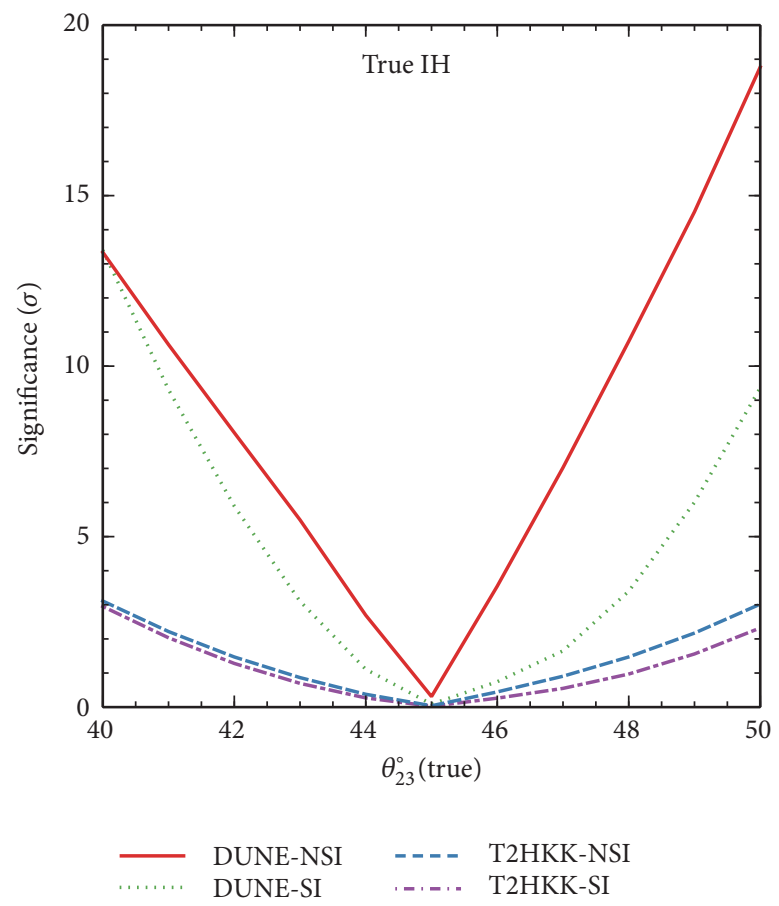

(b)

FIGURE 5: Sensitivity plots for $\theta_{23}$ octant with true NH (left) and true IH (right), assuming NSI parameters $\epsilon_{e \mu}=0.04$ and $\delta_{e \mu}=[-\pi, \pi]$.

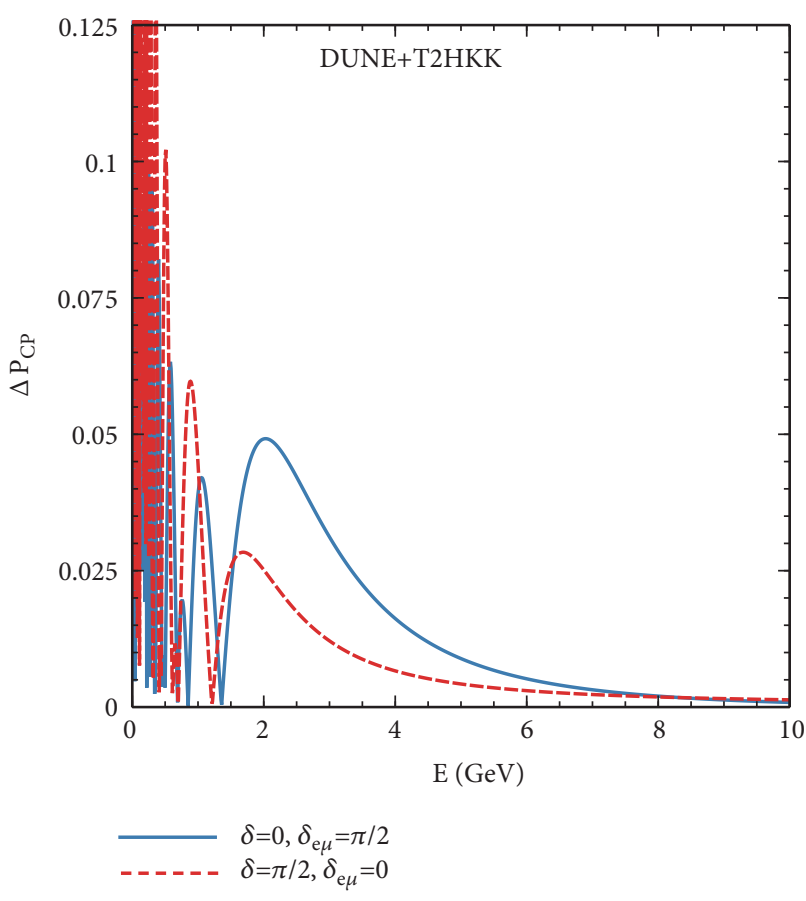

(a)

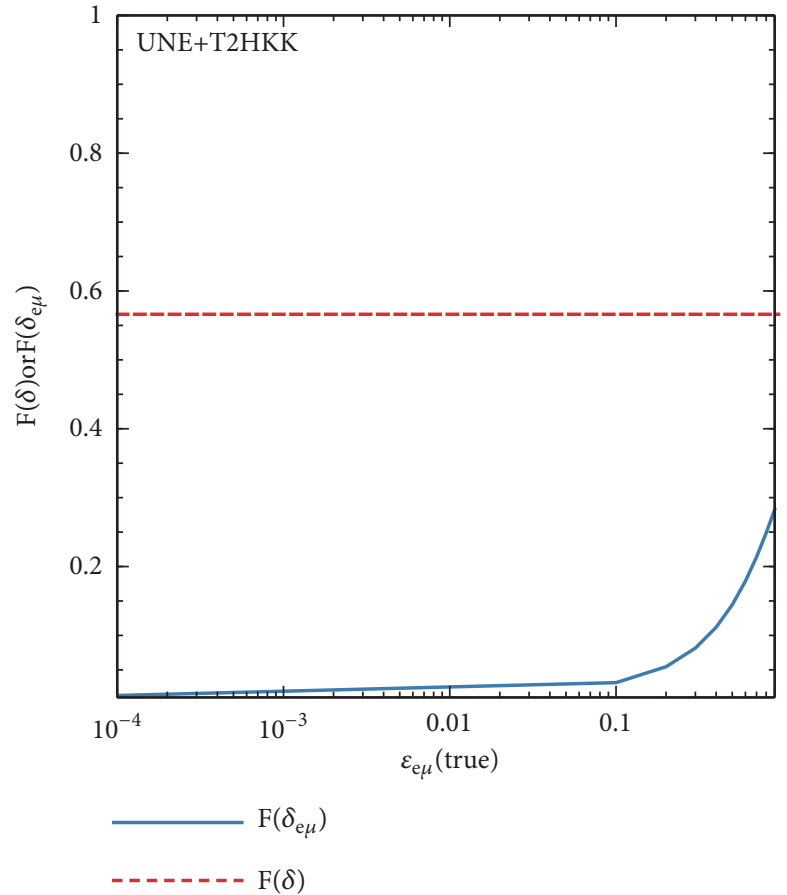

(b)

FIgURE 6: The $C P$ asymmetry as function of neutrino beam energy $E$ for DUNE+T2HKK (left panel). The $C P$ fractions corresponding to $\delta$ and $\delta_{e \mu}$ for which significance $\geq 3 \sigma$ (right panel). 


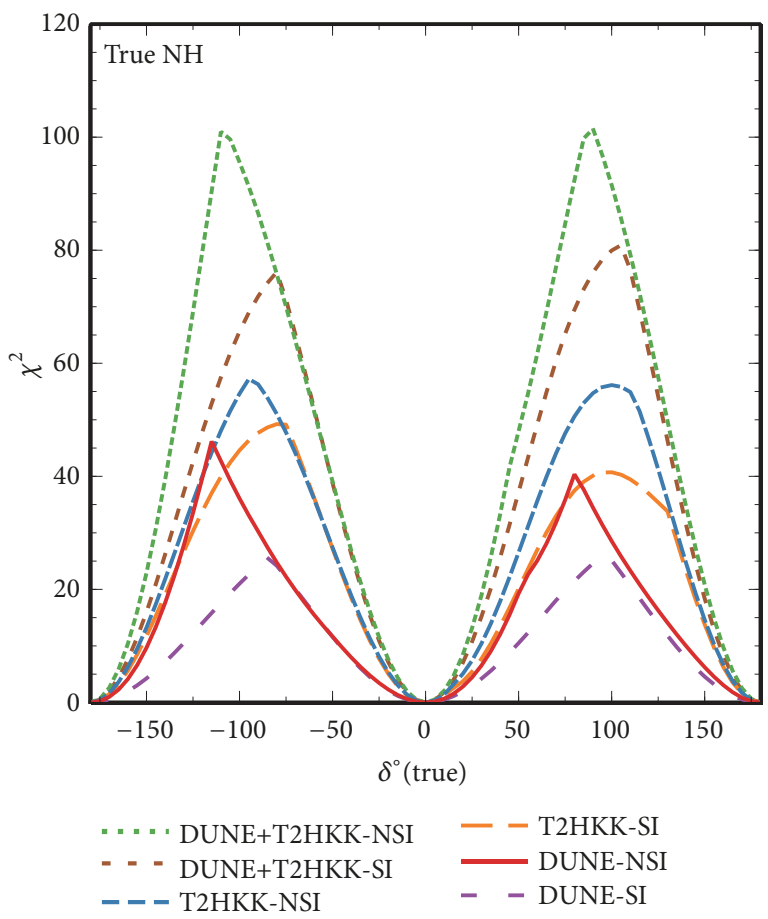

(a)

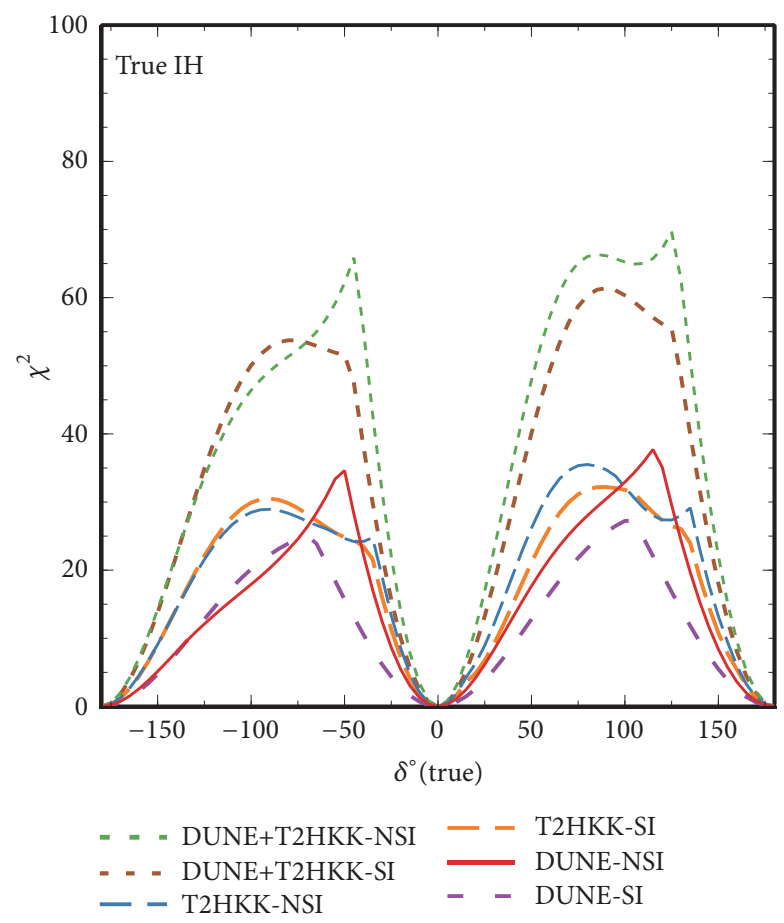

(b)

FIGURE 7: Sensitivity plots for CP violation with true NH (left) and true IH (right) assuming NSI parameters $\epsilon_{e \mu}=0.04$ and $\delta_{e \mu}($ true $)=\pi / 2$.

$\epsilon_{e \mu}$ and corresponding phase to be nonzero (right panel). Furthermore, the future long baseline experiments such as DUNE and T2HKK with higher statistics and better energy resolution, focusing on neutrino beam energy between 1 and $4 \mathrm{GeV}$ energy bracket, may provide better opportunity to resolve mass hierarchy degeneracy (Figures 2(b) and 2(d)). However, for the same energy range DUNE has brighter prospects to resolve the mass degeneracy than T2HKK.

In Figure 3, we have obtained the sensitivity plots for mass hierarchy in presence of $\operatorname{all}\left(\epsilon_{e \mu}, \delta_{e \mu}\right)$ NSI parameters for DUNE and T2HKK in first (second) row with true $\mathrm{NH}$ and true IH. Here, all NSI parameters referred to $\epsilon_{e e}, \epsilon_{e \mu}, \epsilon_{e \tau}, \epsilon_{\mu \mu}, \epsilon_{\mu \tau}, \epsilon_{\tau \tau}$ with corresponding CP phases and their true and test values have been taken from Table 1. For ready reference, we have, also, shown the sensitivity plots for the SI case in Figures 3(e) and 3(f). We find from Figures 3(a) and 3(b) that there is no distinction between true $\mathrm{NH}$ and true $\mathrm{IH}$ sensitivities for any value of $\delta$ (true) at nearly $20 \sigma(3 \sigma)$ C.L. for DUNE (T2HKK) experiments. The hierarchy sensitivity get enhanced when only one NSI parameter $\epsilon_{e \mu}$ and its corresponding $C P$ phase $\delta_{e \mu}$ is present as shown in Figures 3(c) and 3(d). The DUNE experiment in case of true $\mathrm{NH}$ shows stronger hierarchy sensitivity for $-180^{\circ}<\delta<0^{\circ}$ as compared to true $\mathrm{IH}$ and is maximum at $\delta \approx-60^{\circ}(15 \sigma$ C.L. $)$, whereas for $0^{\circ}<\delta<180^{\circ}$ the hierarchy sensitivity in true $\mathrm{IH}$ case is stronger and is maximum at $\delta \approx 40^{\circ}(11 \sigma$ C.L.). For T2HKK experiment the hierarchy sensitivity of true $\mathrm{NH}$ case is stronger than true IH case for the region $-90^{\circ}<\delta<90^{\circ}$ and lies nearly at $5 \sigma$ C.L. It can be noted from Figures 3(a) and 3(b) that $\chi^{2}$ decreases as $\theta_{23}$ changes from lower to higher octant which is in contradistinction to the case with one off-diagonal NSI (Figures 3(c) and 3(d)) and SI case (Figures 3(e) and 3(f)). Consequent to the presence of multiple NSI parameters and long baselines of DUNE and T2HKK, $\theta_{23}$ measurement will be severely affected by the NSI-modified matter effects due to degeneracies between SI and NSI parameters and between NSI parameters. Also, the mass hierarchy sensitivity will be decreased as compared to SI case due to cancellation effects induced by the off-diagonal NSI parameters. In particular, for all NSI parameters, at lower value of $\theta_{23}$ the cancellation effects will be relatively small as compared to SI matter effects which are reflected as increase in the $\chi^{2}$ value. However, cancellation effects will be appreciable for larger value of $\theta_{23}$ resulting in low $\chi^{2}$.

In Figure 4, we have shown octant degeneracy in DUNE and T2HKK with NSI parameters $\epsilon_{e \mu}=0.04$ and $\delta_{e \mu}=$ $[-\pi, \pi]$. It is evident from Figure 4 that octant degeneracy can be resolved with DUNE and T2HKK experiments using combination of neutrino and antineutrino oscillation modes. In Figure 4, the meshed region between solid and dashed lines represents the lower octant (LO) and the forward-diagonal region between dash-dotted and dotted lines represents the higher octant (HO). The left (right) panel in Figure 4 represents neutrino (antineutrino) mode of DUNE and T2HKK experiments. For $\operatorname{LO}\left(\theta_{23}<45^{\circ}\right)$, with $\delta=\pi / 2$ and for $\mathrm{HO}\left(\theta_{23}>45^{\circ}\right)$ with $\delta=-\pi / 2$, both DUNE and T2HKK can resolve the octant degeneracies with neutrino mode only. Moreover, for LO with $\delta=-\pi / 2$ and $\mathrm{HO}$ with $\delta=$ $\pi / 2$, octant degeneracies can be resolved with both DUNE 
and T2HKK with antineutrino mode only. Thus, in general, the neutrino and antineutrino modes are exigent to resolve octant degeneracy in DUNE and T2HKK experiments with matter NSI. Also, we find that the neutrino (antineutrino) beam energy bracket of 1 to $4 \mathrm{GeV}$ can, simultaneously, resolve the mass hierarchy and octant degeneracies in DUNE and T2HKK.

In Figure 5, we have obtained the sensitivity plots of $\theta_{23}$ octant with true NH and true IH for DUNE and T2HKK. It can be seen from Figure 5 that the octant degeneracy can be resolved for both DUNE and T2HKK experiments for true $\mathrm{NH}$ and true $\mathrm{IH}$. The DUNE experiment shows strong sensitivity for $\mathrm{LO}$ in true $\mathrm{NH}$ ( $18 \sigma$ C.L. $)$ and for $\mathrm{HO}$ in true IH (19 $\sigma$ C.L.). The T2HKK experiment has weak sensitivity to resolve octant degeneracy in both true $\mathrm{NH}$ and true $\mathrm{IH}$ case. We have, also, checked that there is not much improvement in $\theta_{23}$ octant sensitivity (over DUNE case) if we take DUNE and T2HKK conjunctively. For comparison, we have, also, shown the SI sensitivity curves of $\theta_{23}$ octant with true $\mathrm{NH}$ and true $\mathrm{IH}$ for DUNE and T2HKK.

The current and future neutrino oscillation experiments are diligently aiming at measuring neutrino mass hierarchy and $C P$ violating phase $\delta$. In presence of NSI (for example, assuming $\epsilon_{e \mu}$ and $\delta_{e \mu}$ nonzero) the situation becomes more complicated due to presence of additional sources of $C P$ violation. The nature may intromit $C P$ violation for wide range of $\delta\left(\delta_{e \mu}\right)$.

In Figure 6(a), we have shown $C P$ asymmetry with neutrino beam energy $E$ for both $\delta$ and $\delta_{e \mu}$ for DUNE+T2HKK. In Figure 6(a), the solid (dashed) line represents $\delta=0, \delta_{e \mu}=$ $\pi / 2\left(\delta=\pi / 2, \delta_{e \mu}=0\right)$ case whereas in Figure $6(\mathrm{~b})$, the solid (dashed) line represents the $C P$ fraction corresponding to the $\delta_{e \mu}(\delta)$. In Figure 6(b), instead of focusing on measurement of $C P$ phases $\left(\delta, \delta_{e \mu}\right)$ we have obtained all possible $\delta$ (true) and $\delta_{e \mu}($ true $)$ values which are different from $C P$ conserving values of $\delta$ and $\delta_{e \mu}$ at $3 \sigma$ C.L. for DUNE+T2HKK. We have shown the effect of real NSI parameter $\left(\epsilon_{e \mu}\right)$ on the discovery reach of $C P$ violation due to $\delta, \delta_{e \mu}$ in Figure 6(b).

For real NSI case $\left(\epsilon_{e \mu} \in\left[10^{-4}, 1.0\right], \delta_{e \mu}=0\right)$, we calculate $C P$ fraction $F(\delta)$, i.e., fraction of $\delta($ true $) \in[-\pi, \pi]$ which is distinguishable from its $C P$ conserving values, at $3 \sigma$ C.L. The parameter space for which significance is less than $3 \sigma$ C.L. has been excluded; thus, $\delta($ test $)$ has been fixed to its $C P$ conserving values $\{0, \pi\}$. The $\chi^{2}$ has been minimized for $\delta$ (true) value from $-\pi$ to $\pi$ and the parameter space for which significance $\geq 3 \sigma$ is considered. It is evident from Figure $6(\mathrm{~b})$ that real NSI parameter $\left(\epsilon_{e \mu}\right)$ has no effect on the discovery reach of $C P$ violation due to $\delta(F(\delta))$, within the available bound on $\epsilon_{e \mu}$, at DUNE+T2HKK.

For complex NSI $\left(\epsilon_{e \mu} \in\left[10^{-4}, 1.0\right], \delta_{e \mu} \neq 0\right)$, with $\delta=0$, i.e., $C P$ violation is only due to NSI phase $\delta_{e \mu}$, we have calculated $C P$ fraction $F\left(\delta_{e \mu}\right)$ on similar lines as that for $F(\delta)$ in real NSI case (replace $\delta$ by $\delta_{e \mu}$ ). It can be seen from Figure 6(b) that $F\left(\delta_{e \mu}\right) \in[0,0.27]$ for $\epsilon_{e \mu} \in\left[10^{-4}, 1.0\right]$. However, for longer baseline $F\left(\delta_{e \mu}\right)$ may be larger even for small value(s) of $\epsilon_{e \mu}$. In this case, if $C P$ violation is not observed at shorter baselines then the larger value of $F\left(\delta_{e \mu}\right)$ implies $C P$ violation due to matter NSI. Also, it is observed from Figure 6(b) that $C P$ fraction $F(\delta)=0.57$ implies that there exist certain range(s) of $\delta$ (true) for which $C P$ violation is undetectable because significance is less than $3 \sigma$. For the case when $C P$ violation is due to both $\delta$ and $\delta_{e \mu}$ and $\delta$ (true) lies below $3 \sigma$ significance, $C P$ violation may be observed for certain range(s) of NSI parameters $\epsilon_{e \mu}$ and $\delta_{e \mu}$. However, it will be difficult to disentangle the source of $C P$ violation i.e., whether it is due to SI phase $\delta$ or NSI phase $\delta_{e \mu}$.

Figure 7 depicts the $C P$ violation sensitivity plots for DUNE, T2HKK, and DUNE+T2HKK experiments with true $\mathrm{NH}$ and true $\mathrm{IH}$. The $\mathrm{CP}$ violation sensitivity of T2HKK is stronger than DUNE in true NH irrespective of the value of $\delta($ true) (Figure $7(\mathrm{a})$ ). For true $\mathrm{IH}, C P$ violation sensitivity of T2HKK is stronger than DUNE except for $25^{\circ}$ range of $\delta$ (true) in $\delta$ (true) $<0$ region and $\delta$ (true) $>0$ region for which $C P$ violation sensitivity of DUNE is stronger than T2HKK. The CP violation sensitivity of DUNE experiment is stronger in true $\mathrm{NH}$ than in true $\mathrm{IH}$ and is maximum at $\delta=-115^{\circ}\left(\delta=115^{\circ}\right)$, in true NH (IH). In combined analysis DUNE+T2HKK, the $C P$ violation sensitivity increases to $10 \sigma$ and $8.2 \sigma$ for true $\mathrm{NH}$ and true $\mathrm{IH}$, respectively. In Figure $7(\mathrm{~b})$, the dip in the T2HKK sensitivity is due to the hierarchy$\delta$ degeneracy. The $\delta$ (true) values at the dip correspond to wrong hierarchy solutions. SI $C P$ violation sensitivity curves for DUNE, T2HKK, and DUNE + T2HKK experiments with true $\mathrm{NH}$ and true $\mathrm{IH}$ have, also, been shown.

\section{Conclusions}

In conclusion, we have investigated the sensitivities of DUNE and T2HKK experiments to resolve mass hierarchy and octant degeneracies in presence of matter NSI. We have, also, studied the $C P$ phase degeneracy due to standard and nonstandard $C P$ phases for DUNE+T2HKK. The results are in consonance with the earlier studies on DUNE. We have analyzed the standard parameter degeneracies in presence of matter NSI for T2HKK experiment. We find that the mass hierarchy degeneracy cannot be resolved in presence of all NSI parameters due to their large experimental uncertainties (Figures 2(a) and 2(c)). However, it can be resolved for neutrino beam energy range 1 to $4 \mathrm{GeV}$ in case of one nonzero NSI parameter $\epsilon_{e \mu}$ and corresponding NSI CP phase $\delta_{e \mu}$ for DUNE and T2HKK experiments (Figures 2(b) and 2(d)).

DUNE and T2HKK show poor sensitivity to resolve mass hierarchy degeneracy in presence of all NSI parameters (Figures 3(a) and 3(b)). However, the sensitivity to mass hierarchy gets enhanced when only one NSI parameter $\epsilon_{\mathrm{e} \mu}$ and its corresponding $C P$ phase $\delta_{e \mu}$ are present (Figures $3(\mathrm{c}$ ) and $3(\mathrm{~d})$ ). DUNE shows stronger hierarchy sensitivity for $-180^{\circ}<\delta<0^{\circ}$ in true $\mathrm{NH}$ than true $\mathrm{IH}$ case with maximum sensitivity at $\delta \approx-60^{\circ}(15 \sigma$ C.L. $)$, whereas for $0^{\circ}<\delta<180^{\circ}$ the hierarchy sensitivity in true IH case is stronger and is maximum at $\delta \approx 40^{\circ}(11 \sigma$ C.L.). T2HKK shows stronger hierarchy sensitivity in true $\mathrm{NH}$ case than true IH case for the region $-90^{\circ}<\delta<90^{\circ}$ and lies nearly at $5 \sigma$ C.L.

Furthermore, for LO $\left(\theta_{23}<45^{\circ}\right)$, with $\delta=\pi / 2$ and for HO $\left(\theta_{23}>45^{\circ}\right)$ with $\delta=-\pi / 2$, both DUNE and T2HKK can resolve the octant degeneracies with neutrino mode only. Moreover, for LO with $\delta=-\pi / 2$ and $\mathrm{HO}$ with $\delta=\pi / 2$, octant 
degeneracies can be resolved with both DUNE and T2HKK with antineutrino mode only. Thus, combination of neutrino and antineutrino mode of DUNE and T2HKK can resolve the octant degeneracy.

The octant degeneracy can be resolved for both DUNE and T2HKK experiments for true NH and true IH (Figure 5). The DUNE experiment shows stronger sensitivity for LO in

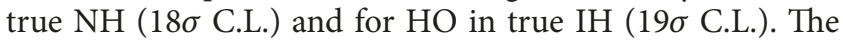
T2HKK experiment has weak sensitivity to resolve octant degeneracy in both true $\mathrm{NH}$ and true $\mathrm{IH}$ case. We have, also, checked that there is not much improvement in $\theta_{23}$ octant sensitivity (over DUNE case) if we take DUNE and T2HKK conjunctively.

The $C P$ asymmetry from nonstandard $C P$ phase $\delta_{e \mu}$ is more than the standard $C P$ phase $\delta$ for neutrino beam energy 1.5 to $7 \mathrm{GeV}$ (Figure 6(a)). From Figure 6(b) we observe that real NSI parameter $\left(\epsilon_{e \mu}\right)$ has no effect on the discovery reach of $C P$ violation due to $\delta(F(\delta)=0.57)$, within the available bound on $\epsilon_{e \mu}$, at DUNE+T2HKK. Also, for complex NSI $\left(\epsilon_{e \mu} \in\left[10^{-4}, 1\right], \delta_{e \mu} \neq 0\right)$ with $\delta=0, F\left(\delta_{e \mu}\right) \in[0,0.27]$ for $\epsilon_{e \mu} \in\left[10^{-4}, 1\right]$. It increases with increase in $\epsilon_{e \mu}$ and is 0.27 when $\epsilon_{e \mu}=1$. Also, it is observed that $F(\delta)=0.57$ implying that there exist certain range(s) of $\delta$ (true) for which $C P$ violation is undetectable because significance is less than $3 \sigma$. For $\delta, \delta_{e \mu} \neq 0$ and $\delta$ (true) lies below $3 \sigma$ significance, then $C P$ violation may be observed for certain range(s) of NSI parameters $\epsilon_{e \mu}$ and $\delta_{e \mu}$. However, it will be difficult to disentangle the source of $C P$ violation, i.e., whether it is due to SI phase $\delta$ or NSI phase $\delta_{e \mu}$.

The $C P$ violation sensitivity of T2HKK is stronger than DUNE in true $\mathrm{NH}$ (Figure $7(\mathrm{a})$ ). However, there exists a $25^{\circ}$ range of $\delta$ (true) in case of true $\mathrm{IH}$ for which $C P$ violation sensitivity of DUNE is stronger than T2HKK. In combined analysis DUNE+T2HKK, the $C P$ violation sensitivity increases to $10 \sigma$ and $8.2 \sigma$ for true $\mathrm{NH}$ and true $\mathrm{IH}$, respectively.

\section{Data Availability}

The data used to support the findings of the present work are taken from $[29,30]$ and are openly accessible.

\section{Conflicts of Interest}

The authors declare that they have no conflicts of interest.

\section{Acknowledgments}

The authors would like to thank Jogesh Rout for valuable discussions during this work. Surender Verma acknowledges the financial support provided by University Grants Commission (UGC), Basic Science Research (BSR), Government of India vide Grant no. F.20-2(03)/2013(BSR). Shankita Bhardwaj acknowledges the financial support provided by the Central University of Himachal Pradesh. The authors, also, acknowledge Department of Physics and Astronomical Science for providing necessary facility to carry out this work.

\section{References}

[1] K. Inoue, "Reactor neutrino oscillation studies with KamLAND," New Journal of Physics, vol. 6, p. 147, 2004.

[2] K. Abe, Y. Hayato, and T. Iida, "Search for differences in oscillation parameters for atmospheric neutrinos and antineutrinos at super-kamiokande," Physical Review Letters, vol. 107, no. 24, Article ID 241801, 2011.

[3] T. Kajita, "Atmospheric neutrinos," New Journal of Physics, vol. 6, p. 194, 2004.

[4] A. B. McDonald, "Solar neutrinos," New Journal of Physics, vol. 6, p. 121, 2004.

[5] F. P. An, J. Z. Bai, and A. B. Balantekin, "Observation of electronantineutrino disappearance at daya bay," Physical Review Letters, vol. 108, Article ID 171803, 2012.

[6] A. M. Gago, H. Minakata, H. Nunokawa, S. Uchinami, and R. Zukanovich, "Zukanovich Funchal," JHEP, vol. 049, p. 1001, 2010.

[7] J. Liao, D. Marfatia, and K. Whisnant, "Degeneracies in longbaseline neutrino experiments from nonstandard interactions," Physical Review D: Particles, Fields, Gravitation and Cosmology, vol. 93, no. 9, Article ID 093016, 2016.

[8] S. Fukasawa, M. Ghosh, and O. Yasuda, "Complementarity between Hyperkamiokande and DUNE in determining neutrino oscillation parameters," Nuclear Physics B, vol. 918, pp. 337-357, 2017.

[9] T. Ohlsson, "Status of non-standard neutrino interactions," Reports on Progress in Physics, vol. 76, no. 4, Article ID 044201, 2013.

[10] O. G. Miranda and H. Nunokawa, "Non standard neutrino interactions: current status and future prospects," New Journal of Physics, vol. 17, Article ID 095002, 2015.

[11] L. Wolfenstein, "Neutrino oscillations in matter," Physical Review D: Particles, Fields, Gravitation and Cosmology, vol. 17, p. 2369, 1978.

[12] K. Abe, H. Aihara, C. Andreopoulos et al., "A Long Baseline Neutrino Oscillation Experiment Using J-PARC Neutrino Beam and Hyper-Kamiokande," https://arxiv.org/abs/1412.4673.

[13] R. Acciarri, M. A. Acero, M. Adamowski et al., "Long-Baseline neutrino facility (LBNF) and deep underground neutrino experiment (DUNE) conceptual design report volume 2: the physics program for DUNE at LBNF," https://arxiv.org/abs/1512 .06148 .

[14] K. Abe, K. E. Abe, S. H. Ahn et al., "Physics potentials with the second hyper-Kamiokande detector in Korea," Progress of Theoretical and Experimental Physics, Article ID 063C01, p. 65, 2018.

[15] C. Biggio, M. Blennow, and E. Fernández-Martínez, "General bounds on non-standard neutrino interactions," Journal of High Energy Physics, vol. 2009, no. 08, p. 090, 2009.

[16] S. Choubey and T. Ohlsson, "Bounds on non-standard neutrino interactions using PINGU," Physics Letters B, vol. 739, pp. 357364, 2014.

[17] M. M. Guzzo, A. Masiero, and S. T. Petcov, "On the MSW effect with massless neutrinos and no mixing in the vacuum," Physics Letters B, vol. 260, no. 1-2, pp. 154-160, 1991.

[18] M. Masud and P. Mehta, "Nonstandard interactions and resolving the ordering of neutrino masses at DUNE and other long baseline experiments," Physical Review D: covering particles, fields, gravitation, and cosmology, vol. 94, Article ID 053007, 2016. 
[19] M. Fukugita and T. Yanagida, "Barygenesis without grand unification," Physics Letters B, vol. 174, no. 1, pp. 45-47, 1986.

[20] S. Davidson, E. Nardi, and Y. Nir, "Leptogenesis," Physics Reports, vol. 466, no. 4-5, pp. 105-177, 2008.

[21] J. Liao, D. Marfatia, and K. Whisnant, "Nonstandard neutrino interactions at DUNE, T2HK and T2HKK," Journal of High Energy Physics, vol. 2017, p. 71, 2017.

[22] K. N. Deepthi, S. Goswami, and N. Nath, "Challenges posed by non-standard neutrino interactions in the determination of $\delta_{C P}$ at DUNE," Nuclear Physics B, vol. 936, pp. 91-105, 2018.

[23] M. Masud and P. Mehta, "Nonstandard interactions spoiling the CP violation sensitivity at DUNE and other long baseline experiments," Physical Review D, vol. 94, Article ID 013014, 2016.

[24] M. Masud, A. Chatterjee, and P. Mehta, "Probing the CP violation signal at DUNE in the presence of non-standard neutrino interactions," Journal of Physics G: Nuclear and Particle Physics, vol. 43, no. 9, Article ID 095005, 2016.

[25] K. N. Deepthi, S. Goswami, and N. Nath, "Can nonstandard interactions jeopardize the hierarchy sensitivity of DUNE?" Physical Review D, vol. 96, Article ID 075023, 2017.

[26] S. K. Agarwalla, S. S. Chatterjee, and A. Palazzo, "Degeneracy between $\theta_{23}$ octant and neutrino non-standard interactions at DUNE," Physics Letters B, vol. 762, pp. 64-71, 2016.

[27] S. K. Raut, "Matter effects at the T2HK and T2HKK experiments," Physical Review D: Particles, Fields, Gravitation and Cosmology, vol. 96, Article ID 075029, 2017.

[28] P. Huber, M. Lindner, and W. Winter, "Simulation of longbaseline neutrino oscillation experiments with GLoBES: (General Long Baseline Experiment Simulator)," Computer Physics Communications, vol. 167, no. 3, pp. 195-202, 2005.

[29] P. Huber, J. Kopp, M. Lindner, M. Rolinec, and W. Winter, "New features in the simulation of neutrino oscillation experiments with GLoBES 3.0: (General Long Baseline Experiment Simulator)," Computer Physics Communications, vol. 177, pp. 432-438, 2007.

[30] M. C. Gonzalez-Garcia, M. Maltoni, and T. Schwetz, "Updated fit to three neutrino mixing: status of leptonic CP violation," Journal of High Energy Physics, vol. 2014, no. 52, p. 1411, 2014.

[31] http://home.fnal.gov/ ljf26/DUNE2015CDRFluxes.

[32] Z. Rahman, A. Dasgupta, and R. Adhikari, "The discovery reach of CP violation in neutrino oscillation with non-standard interaction effects," Journal of Physics G: Nuclear and Particle Physics, vol. 42, no. 6, Article ID 065001, 2015. 

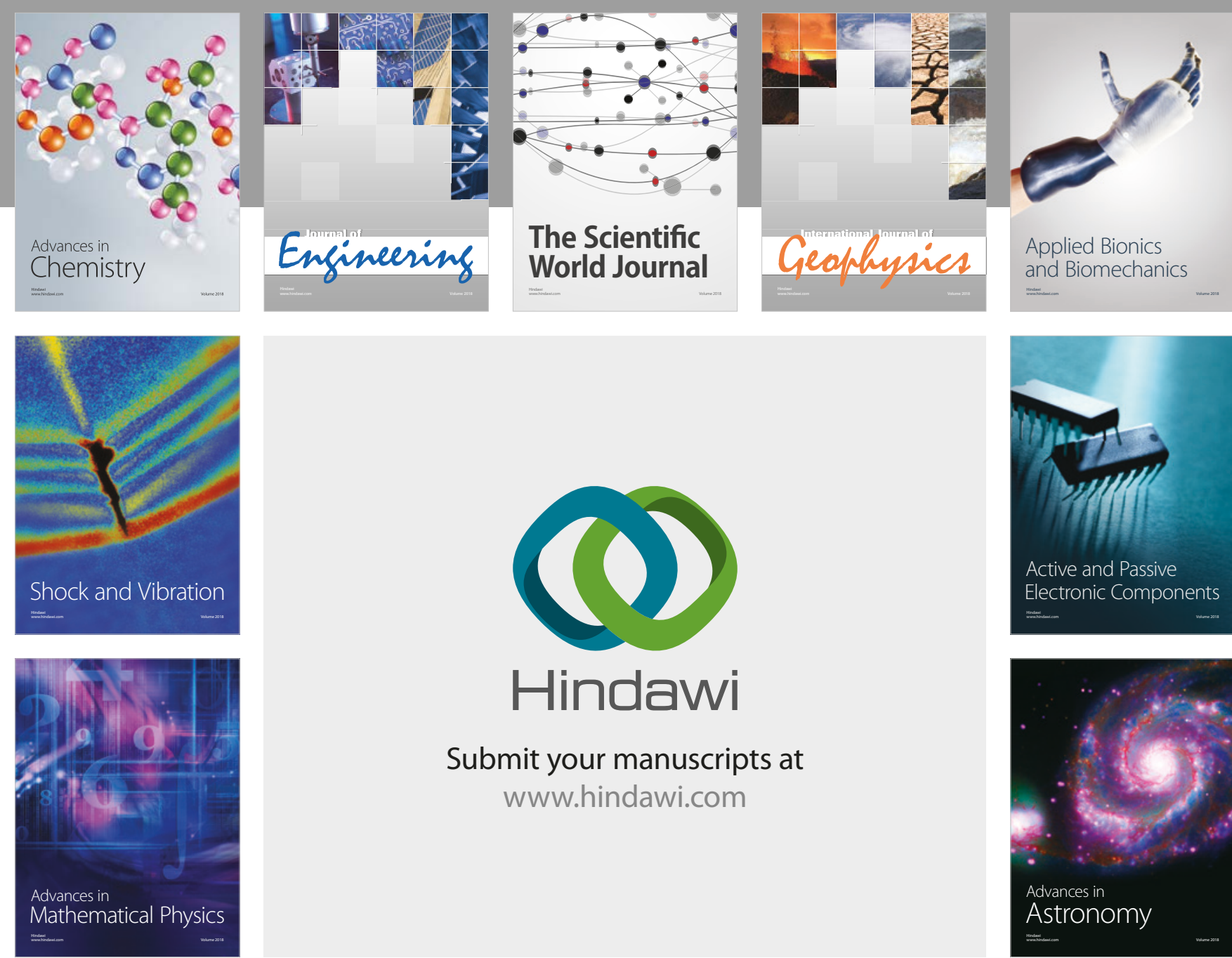

Submit your manuscripts at

www.hindawi.com

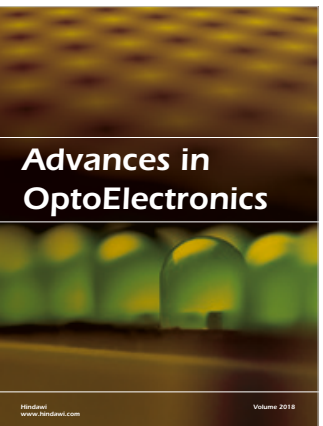

\section{Rotcting Machinery}
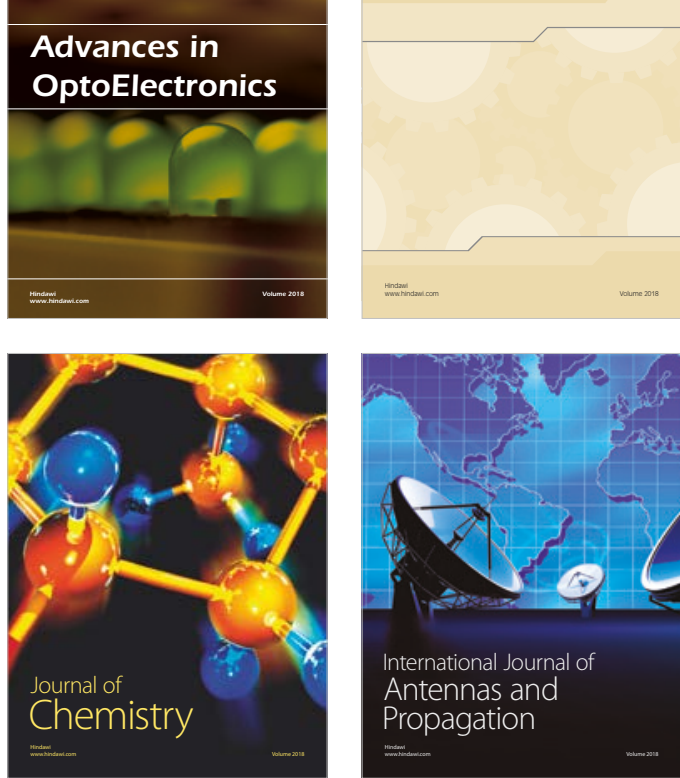

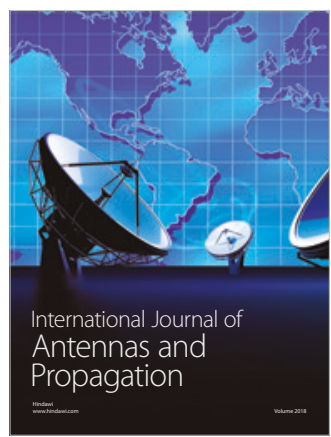

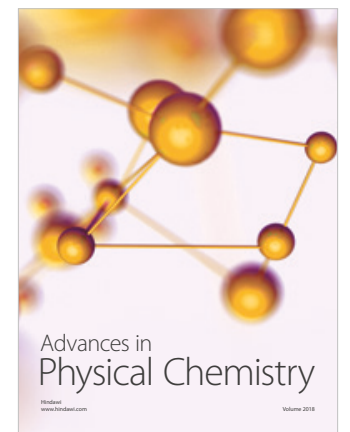

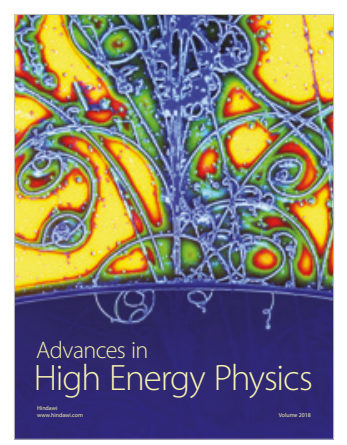

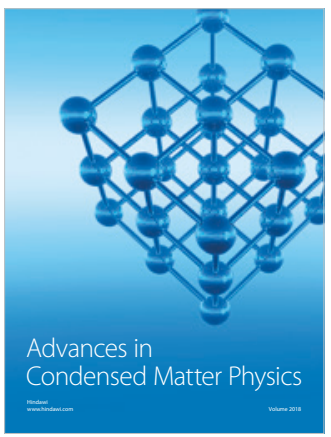

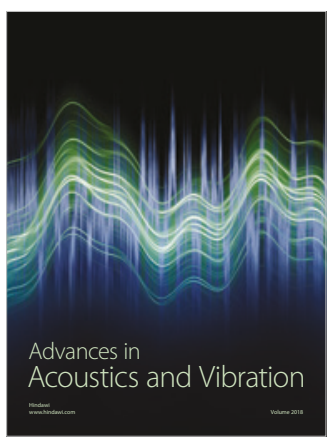

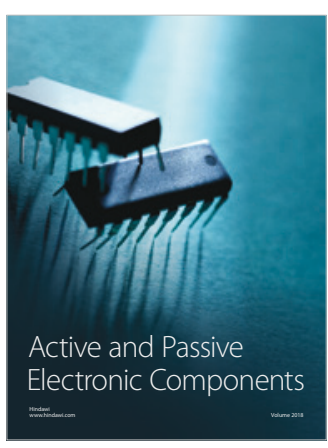
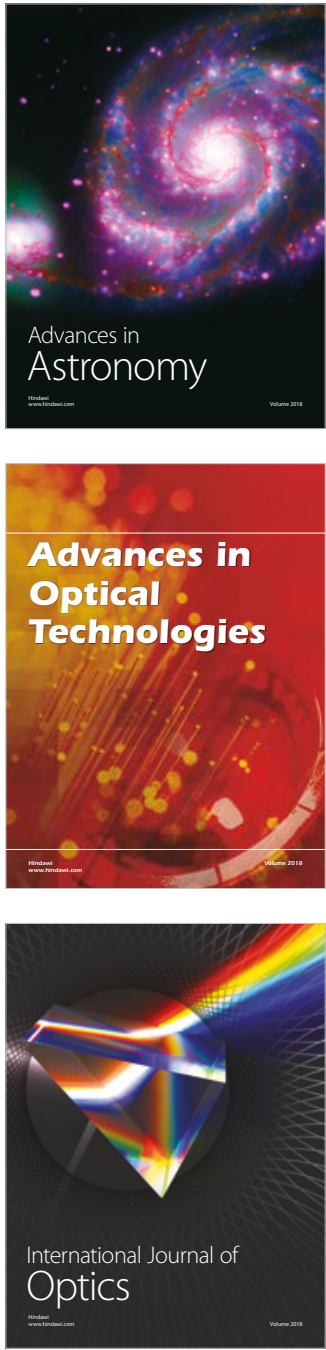